\title{
PERTURBED DYNAMICAL SYSTEMS WITH AN ATTRACTING SINGULARITY AND WEAK VISCOSITY LIMITS IN HAMILTON-JACOBI EQUATIONS
}

\author{
B. PERTHAME
}

\begin{abstract}
We give a new PDE proof of the Wentzell-Freidlin theorem concerning small perturbations of a dynamical system

$$
\begin{aligned}
& L_{\varepsilon} u_{\varepsilon}=-\frac{\varepsilon}{2} \Delta u_{\varepsilon}-b \cdot \nabla u_{\varepsilon}=0 \text { in } \Omega, \\
& u_{\varepsilon}=\varphi \quad \text { on } \partial \Omega .
\end{aligned}
$$

We prove that, if $b$ has a single attractive singular point, $u_{\varepsilon}$ converges uniformly on compact subsets of $\Omega$, and with an exponential decay, to a constant $\mu$, and we determine $\mu$. We also treat the case of Neumann boundary condition. In order to do so, we perform the asymptotic analysis for some ergodic measure which leads to a study of the viscosity limit of a Hamilton-Jacobi equation. This is achieved under very general assumptions by using a weak formulation of the viscosity limits of these equations.
\end{abstract}

RÉSUMÉ. Nous donnons une nouvelle preuve, par des méthodes EDP, du théorème de Wentzell-Freidlin concernant les petites perturbations d'un système dynamique:

$$
\begin{aligned}
& L_{\varepsilon} u_{\varepsilon}=-\frac{\varepsilon}{2} \Delta u_{\varepsilon}-b \cdot \nabla u_{\varepsilon}=0 \text { dans } \Omega, \\
& u_{\varepsilon}=\varphi \quad \text { sur } \partial \Omega .
\end{aligned}
$$

Nous prouvons que, si $b$ a un seul point singulier attractif, alors $u_{\varepsilon}$ converge vers une constant $\mu$, uniformément sur tout compact, et avec une vitesse exponentielle. Nous déterminons $\mu$. Nous traitons aussi le cas de conditions aux limites de Neuman. Pour cela, nous faisons l'analyse asymptotique d'une mesure ergodique intervenant naturellement dans le problème, ce qui revient à étudier la limite par viscosité évanescente dans une équation de HamiltonJacobi. Ceci est réalisé sous des hypothèses très générales gâce à un passage à la limite faible dans cette équation.

In this paper, we present some new ideas to simplify and generalize the PDE proof of the result of Wentzell-Freidlin $[14,26]$ concerning small random perturbations of a dynamical system with a singular point of attracting type. The problem can be described as follows. Let $u_{\varepsilon}$ solve

$$
\begin{aligned}
& L_{\varepsilon} u_{\varepsilon}=-\frac{\varepsilon}{2} \Delta u_{\varepsilon}-b \cdot \nabla u_{\varepsilon}=0 \text { in } \Omega, \\
& u_{\varepsilon}=\varphi \quad \text { on } \partial \Omega,
\end{aligned}
$$

Received by the editors November 27, 1987 and, in revised form, June 6, 1988.

1980 Mathematics Subject Classification (1985 Revision). Primary 34E20, 35B25, 35F30, 49A22.

Key words and phrases. Dynamical systems, singular perturbations, Hamilton-Jacobi equations, viscosity limit, ergodic measure, large deviations. 
and let $b$ be of attracting type (i.e. any trajectory of $\dot{y}_{t}=b\left(y_{t}\right), y(0)=x \in \bar{\Omega}$, tends to 0 as $t$ tends to infinity). Then, it is known that $u_{\varepsilon}$ converges to some constant $\mu$ as $\varepsilon$ tends to 0 and the problem is to determine $\mu$. This has been achieved first by Wentzell-Freidlin (loc. cit.) using probabilistic arguments and they show that $\mu$ may be written

$$
\mu=\int_{\partial \Omega} \varphi(x) d P(x),
$$

where the probability measure $P$ is supported by the set of minimizing points on $\partial \Omega$ of the so-called quasi-potential function

$$
\begin{gathered}
Q(x)=\operatorname{Inf}\left\{\int_{0}^{\infty} L(y(s), \dot{y}(s)) d s ; y \in H^{1}\left(\mathbf{R}^{+}\right), y(0)=x, y(\cdot) \in \bar{\Omega}\right\}, \\
L(y, v)=|v+b(y)|^{2} / 2 .
\end{gathered}
$$

The proof relies on the large deviations principle. Recently Day [8] has given an exact formula for $P$, namely he has proved the following formula, given earlier by Matkowsky and Schuss [23],

$$
\mu=\lim _{\varepsilon \rightarrow 0}\left(\int_{\partial \Omega} \varphi b \cdot n R e^{-Q / \varepsilon}\right) /\left(\int_{\partial \Omega} b \cdot n R e^{-Q / \varepsilon}\right),
$$

where $R$ solves a linear first order equation (the main difficulty being to give a sense to this equation which coefficients are not smooth). We refer to [8] for the numerous historical developments about this problem, and we restrict ourself to the particular situation we are interested in, namely the PDE approach.

From a purely PDE point of view, Kamin [19] has proved that $u_{\varepsilon}$ converges to a constant. But for the determination of this constant, although the general formula has been formally derived for a few years in [23], the only rigorous result which is known is the case when $Q$ has a unique minimum point $P_{0}$ on $\partial \Omega$. Then Kamin [20] has proved that the measure $P$ in (2) is a Dirac mass at $P_{0}$. The function $Q$ appears, in the above papers, as a particular solution of the Hamilton-Jacobi (H.J. in short) equation

$$
|\nabla V|^{2} / 2+(b, \nabla V)=0 \text { in } \Omega \text {. }
$$

Here, we intend to give a completely new proof of the convergence of $u_{\varepsilon}$ toward a constant and we will prove, using only this H.J. equation, that $P$ is supported by the set of minimum points of the solution of some boundary value problem associated to (5), namely the state constraints problem, and we will make clear that its unique solution is given by (3). We introduce two main ideas. The first one is that the Wentzell-Freidlin Theorem may be reduced to the asymptotic analysis of the ergodic measure given by $(6)-\left(6^{\prime}\right)$ below. The second idea is that we may perform this analysis, under very weak assumptions on $b$, using the new weak limit introduced in Barles and Perthame [2,3], already used by Ishii [17]. Let us mention also that it is classical to treat problems of large deviations type by PDE arguments, using in particular the method developed 
recently by Fleming and Souganidis [13] and Evans and Ishii [9]; see also Evans and Souganidis [10,11] and Bardi [1].

The importance of the ergodic measure for this problem can be seen from the determination of $\mu$ that we explain now. Consider, following the method introduced by Matkowsky and Schuss [23], the adjoint problem associated to (1),

$$
L_{\varepsilon}^{*} v_{\varepsilon}=-\frac{\varepsilon}{2} \Delta v_{\varepsilon}+\operatorname{div}\left(b v_{\varepsilon}\right)=0 \text { in } \Omega ;
$$

then set $v_{\varepsilon}=e^{-V_{\varepsilon} / \varepsilon}$. A simple integration by parts gives

$$
\begin{aligned}
0 & =\int_{\Omega} v_{\varepsilon} L_{\varepsilon} u_{\varepsilon}-u_{\varepsilon} L_{\varepsilon}^{*} v_{\varepsilon} \\
& =\int_{\partial \Omega}-\frac{\varepsilon}{2} v_{\varepsilon} \partial u_{\varepsilon} / \partial n+\left\{\frac{\varepsilon}{2} \partial v_{\varepsilon} / \partial n-b \cdot n v_{\varepsilon}\right\} u_{\varepsilon} .
\end{aligned}
$$

But Kamin [19] has proved (see also our new proof in $\S I I I)$ that on $\partial \Omega$ we have

$$
\frac{\varepsilon}{2} \partial u_{\varepsilon} / \partial n+\left(\varphi-u_{\varepsilon}(0)\right) b \cdot n=\rho(\varepsilon) \rightarrow 0 \quad \text { as } \varepsilon \rightarrow 0 \text { (uniformly). }
$$

Therefore, we obtain

$$
0=\int_{\partial \Omega}\left[\rho(\varepsilon)-\left(\varphi-u_{\varepsilon}(0)\right) b \cdot n\right] e^{-V_{\varepsilon} / \varepsilon}+\varphi\left[\frac{\varepsilon}{2} \partial v_{\varepsilon} / \partial n-b \cdot n v_{\varepsilon}\right],
$$

and thus the problem is now to determine the behavior of $V_{\varepsilon}$ or $v_{\varepsilon}$ as $\varepsilon$ goes to 0 . Different tricks are used in the above cited papers to perform this, but no direct relation has been established between (6), (5) and (3).

Let us give a new understanding of the boundary conditions associated to (5) and (6) so that the determination of $\mu$ from (8) becomes very simple and the relation between (6), (5) and (3) becomes clear. We add to (6) the boundary condition

$$
\frac{\varepsilon}{2} \partial v_{\varepsilon} / \partial n-b \cdot n v_{\varepsilon}=0 \text { on } \partial \Omega .
$$

The problem $(6)-\left(6^{\prime}\right)$ is classical, its solution is the ergodic measure (choose $\int_{\Omega} v_{\varepsilon} d x=1$ ) associated to the reflected diffusion with drift $b$. In particular, it is shown that $v_{\varepsilon}$ remains positive and thus the exponential transformation is possible and $V_{\varepsilon}$ satisfies

$$
\begin{gathered}
-\frac{\varepsilon}{2} \Delta V_{\varepsilon}+\left|\nabla V_{\varepsilon}\right|^{2} / 2+\left(b, \nabla V_{\varepsilon}\right)=\varepsilon \operatorname{div} b \text { in } \Omega, \\
\partial V_{\varepsilon} / \partial n=-2 b \cdot n \text { on } \partial \Omega .
\end{gathered}
$$

The second new idea that we will develop below is that $V_{\varepsilon}$ is bounded in $L^{\infty}$ uniformly as $\varepsilon$ tends to 0 and that $V_{\varepsilon}$ converges uniformly to the solution $V_{0}$ of (5) with the boundary condition

$$
|\nabla V|^{2} / 2+(b, \nabla V) \geq 0 \text { on } \partial \Omega \text {. }
$$

This boundary condition, which has been introduced by Soner [25], has to be understood in the viscosity sense of Crandall and Lions [7]. The point is that 
$Q$ still satisfies $(5)-\left(5^{\prime}\right)$ and a uniqueness result holds for $(5)-\left(5^{\prime}\right)$. Namely, we will prove that $V_{\varepsilon}$ is continuous at the attraction point (say 0 ) and since we may add a constant to $V_{\varepsilon}$, we have $V_{\varepsilon}(0)=V(0)=0$. Now $(5)-\left(5^{\prime}\right)$ admits a unique viscosity solution which is continuous at 0 and which value at 0 is 0 and thus it is given by (3). Let us only point out that $V \equiv 0$ does not satisfy $\left(5^{\prime}\right)$ in the viscosity sense described in Appendix 1 (see Appendix 1 for a proof). In fact $\left(5^{\prime}\right)$ is associated with optimal control problems with state constraints (see [25] and Capuzzo-Dolcetta and Lions [6]). This means that $\left(5^{\prime}\right)$ is naturally associated with the constraint $y(\cdot) \in \bar{\Omega}$ in (3).

Putting together these results, we get from $(8)$ and $\left(6^{\prime}\right)$

$$
\begin{aligned}
u_{\varepsilon}(0)= & \left(\int_{\partial \Omega} \rho(\varepsilon) e^{-V_{\varepsilon} / \varepsilon}\right) /\left(\int_{\partial \Omega} b \cdot n e^{-V_{\varepsilon} / \varepsilon}\right) \\
& +\left(\int_{\partial \Omega} \varphi b \cdot n e^{-V_{\varepsilon} / \varepsilon}\right) /\left(\int_{\partial \Omega} b \cdot n e^{-V_{\varepsilon} / \varepsilon}\right),
\end{aligned}
$$

i.e.

$$
\begin{gathered}
\mu_{\varepsilon}=\left(\int_{\partial \Omega} \varphi b \cdot n e^{-V_{\varepsilon} / \varepsilon}\right) /\left(\int_{\partial \Omega} b \cdot n e^{-V_{\varepsilon} / \varepsilon}\right), \\
\mu=\lim _{\varepsilon \rightarrow 0} u_{\varepsilon}(0)=\lim _{\varepsilon \rightarrow 0} \mu_{\varepsilon},
\end{gathered}
$$

and, since $V_{\varepsilon} \rightarrow V_{0}$ in $C(\bar{\Omega}), V_{0}$ a solution of $(5)-\left(5^{\prime}\right)$, we obtain the WentzellFreidlin result. Indeed, it is very simple to see that (10) implies that the measure $P$ in (2) is supported by the set of minimum points of $V_{0}$, partitioning $\partial \Omega$ into two subsets, a neighborhood of the minimum points of $V_{0}$ and its complementary set.

This paper is organized as follows. In $\S \mathbf{I}$, we state precisely our results and assumptions. In $\S I I$, we prove our results concerning the asymptotic behavior of the ergodic measure. In the last section, we prove the convergence of $u_{\varepsilon}$ and we perform the boundary layer analysis necessary to establish (7). We recall the basic definitions about viscosity solutions of H.J. equations in Appendix 1 .

\section{Assumptions AND MAIN Result}

From the introduction, the determination of the support of $P$ is reduced to prove that there exists a positive solution $v_{\varepsilon}$ to (9) and that it converges uniformly to a solution of $(5)-\left(5^{\prime}\right)$. We state precisely these results below. Here $\Omega$ denotes a smooth $\left(C^{2}\right)$ bounded open subset of $\mathbf{R}^{N}$, and $n(x)$ denotes the unit outward normal to $\partial \Omega$ at $x$. Our first result concerns the existence of $v_{\varepsilon}$.

Theorem 1. Let $b$ belong to $L^{\infty}(\Omega)$. Then there exists a unique variational solution $v_{\varepsilon}=W^{1, p}(\Omega) \cap C(\bar{\Omega}), 1 \leq p<\infty$, to

$$
\begin{gathered}
-\frac{\varepsilon}{2} \Delta v_{\varepsilon}+\operatorname{div}\left(b v_{\varepsilon}\right)=0 \quad \text { in } \Omega, \underset{\bar{\Omega}}{\operatorname{Max}} v_{\varepsilon}=1, \\
\frac{\varepsilon}{2} \partial v_{\varepsilon} / \partial n-b \cdot n v_{\varepsilon}=0 \quad \text { on } \partial \Omega .
\end{gathered}
$$


Moreover, one has for some $C$ independent of $\varepsilon$,

$$
e^{-C / \varepsilon} \leq v_{\varepsilon} \leq 1 \text {. }
$$

We will discuss this result later. Let us now give some assumptions in order to study the convergence of $\varepsilon \log v_{\varepsilon}$. We assume

(H2) $b \in C(\bar{\Omega} \backslash\{0\}), \quad b \in L^{\infty}(\Omega), \quad \operatorname{div} b \in L^{\infty}(\Omega), \quad b \cdot n \leq 0 \quad$ on $\partial \Omega$,

(H2) For $\delta>0$ small enough, there exists a function

$$
\psi_{\delta} \in W^{1, \infty}\left(\Omega \backslash \bar{B}_{\delta}\right)
$$

satisfying for some $\rho(\delta)>0$

$$
\begin{array}{ll}
\left|\nabla \psi_{\delta}\right|^{2} / 2+\left(b, \nabla \psi_{\delta}\right) \leq-\rho(\delta) & \text { in } \Omega \backslash \bar{B}_{\delta}, \\
\partial \psi_{\delta} / \partial n \leq-2 b \cdot n & \text { on } \partial \Omega, \\
\psi_{\delta}=0 & \text { on } \partial B_{\delta} .
\end{array}
$$

Here the inequalities have to be understood in the viscosity sense of CrandallLions (loc. cit.) and we refer the reader to Appendix 1 for a definition of viscosity solutions to H.J. equations.

In order to be more specific, let us give a sufficient condition for $(\mathrm{H} 2)$ to hold.

$$
\begin{aligned}
& b \text { is continuous in } \bar{\Omega} \backslash\{0\} \text { and } \forall \delta>0, \exists T(\delta)<+\infty, \forall x \in \\
& \bar{\Omega} \backslash B_{\delta}, \forall y(\cdot) \text { satisfying }
\end{aligned}
$$

$$
\dot{y}(s)=-b(y(s)), \quad y(0)=x,
$$

then,

$$
\exists s, 0 \leq s \leq T_{\delta}, \quad y(s) \notin \bar{\Omega} \backslash B_{\delta} .
$$

Here, as in (H2), $B_{\delta}$ denotes the ball of center 0 and radius $\delta$. The proof that $(\mathrm{H} 3) \Rightarrow(\mathrm{H} 2)$ is given in Appendix 2 .

We may now state our main result.

Theorem 2. Under assumptions $(\mathrm{H} 1)$ and $(\mathrm{H} 2), V_{\varepsilon}=-\varepsilon \log v_{\varepsilon}$ converges uniformly in $\bar{\Omega}$ to $V_{0} \in C(\bar{\Omega})$, the unique continuous viscosity solution of

$$
\begin{gathered}
|\nabla V|^{2} / 2+(b, \nabla V)=0 \quad \text { in } \Omega \backslash\{0\}, \\
|\nabla V|^{2} / 2+(b, \nabla V)>0 \quad \text { on } \partial \Omega,
\end{gathered}
$$

such that $V(0)=0$.

Let us comment on Theorems 1 and 2. First, as we pointed it out in the introduction, the existence of $v_{\varepsilon}$ is classical since $v_{\varepsilon}$ represents (up to a multiplication by a constant) the ergodic measure associated with some reflected diffusion process (the adjoint of $(6)-\left(6^{\prime}\right)$ is a pure Neumann problem for which 0 is the first eigenvalue). The proof of the existence of a positive solution to $(6)-\left(6^{\prime}\right)$ can be found in Bensoussan [4] for $b \in L^{\infty}$ or Bensoussan and Lions 
[5] for $b \in C^{1}$. The new point here is the explicit lower bound (11) that we obtain through the Harnack Inequality. Let us recall that $(6)-\left(6^{\prime}\right)$ holds in a variational sense and a correct formulation is

$$
\frac{\varepsilon}{2} \int_{\Omega} \nabla v_{\varepsilon} \cdot \nabla v-\int_{\Omega} v_{\varepsilon} b \cdot \nabla v=0 \quad \forall v \in H^{1}(\Omega) .
$$

The result of Theorem 2 follows the idea of Fleming [12] to study the asymptotic behavior of $v_{\varepsilon}$ by a log-transformation. Let us only point out that this method leads generally to H.J. equations and has been studied by various authors (see the references in the introduction). The new point here is that we will perform a very weak passage to the limit as $\varepsilon \rightarrow 0$ so that we do not use estimates on $\nabla v_{\varepsilon}$ or compactness in $C(\bar{\Omega})$. This allows us to deal with very general assumptions on $b$ and to get rid of any regularity on $V_{0}$.

Finally, $(5)-\left(5^{\prime}\right)$ is associated with a state constraint $[6,25]$ control problem which can be described as follows. The Hamiltonian in (5) is given by

$$
H(x, p)=|p|^{2} / 2+(b(x), p)=\sup _{v \in \mathbf{R}^{N}}\{-(v, p)-L(x, v)\} .
$$

Therefore, $V_{0}$ is given through the following procedure; let $y_{x}(\cdot)$ satisfy for some measurable $v(\cdot)$

$$
d y_{x}(t)=v(t) d t, \quad y_{x}(0)=x
$$

then

$$
V_{0}(x)=\inf \left\{\int_{0}^{\infty} L\left(y_{x}(t), v(t)\right) d t ; y_{x}(s) \in \bar{\Omega} \forall s \geq 0\right\},
$$

which is equivalent to formula (3). This also explains why the boundary condition $\left(5^{\prime}\right)$ holds. The natural one should be

$$
\partial V_{0} / \partial n=-2 b \cdot n,
$$

as it is for $V_{\varepsilon}$. But the costs to reflect $(-2 b \cdot n)$ is too expensive and this boundary condition is lost through a boundary layer effect and gives $\left(5^{\prime}\right)$.

The proofs of Theorems 1 and 2 are given in $\S I I$.

Finally, let us state our results on the behavior of $u_{\varepsilon}$, and on the Neumann problem.

Theorem 3. Under assumptions $(\mathrm{H} 1)$ and $(\mathrm{H} 2)$, let $\varphi \in C(\partial \Omega)$ and let $u_{\varepsilon}$ solve

$$
\left\{\begin{array}{l}
L_{\varepsilon} u_{\varepsilon}=-\frac{\varepsilon}{2} \Delta u_{\varepsilon}-b \cdot \nabla u_{\varepsilon}=0, \quad u_{\varepsilon} \in C^{2}(\Omega) \cap C(\bar{\Omega}), \\
u_{\varepsilon}=\varphi \text { on } \partial \Omega .
\end{array}\right.
$$

Then, $u_{\varepsilon}-u_{\varepsilon}(0)$ converges uniformly on every compact subset of $\Omega$ to 0 , and with an exponential rate if $\varphi \in C^{1, \beta}(\partial \Omega)$ for some $\beta>0$. If $b \cdot n<0$ on $\partial \Omega$, then $u_{\varepsilon}(0)-\mu_{\varepsilon}$ converges to 0 (where $\mu_{\varepsilon}$ is given by (10)), and if $\varphi \in C^{1, \beta}(\partial \Omega)$ then (7) holds. 
Theorem 4. Under assumptions $(\mathrm{H} 1)$ and $(\mathrm{H} 2)$, let $\varphi \in C(\partial \Omega)$, let $b \cdot n<0$ on $\partial \Omega$, and let $z_{\varepsilon}$ solve

$$
\begin{aligned}
& L_{\varepsilon} z_{\varepsilon}=-\frac{\varepsilon}{2} \Delta z_{\varepsilon}-b \cdot \nabla z_{\varepsilon}=0, \quad z_{\varepsilon} \in H^{1}(\Omega) \cap L^{\infty}(\Omega), \\
& \frac{\varepsilon}{2} \partial z_{\varepsilon} / \partial n=-b \cdot n\left(\varphi-\mu_{\varepsilon}\right) \quad \text { on } \partial \Omega, \quad \int_{\Omega} v_{\varepsilon} z_{\varepsilon} d x=0
\end{aligned}
$$

(where $\mu_{\varepsilon}$ is given by (10)). Then $z_{\varepsilon}$ remains uniformly bounded and converges uniformly to 0 on every compact subset of $\Omega$ and with an exponential rate.

The restriction $b \cdot n<0$ in Theorem 3 needs to be made for technical reasons: we need it to perform a part of the boundary layer analysis necessary to prove (7). In Theorem 4 it seems more fundamental to get uniform bounds on $z_{\varepsilon}$ (since solutions of (1) are approximately solutions of the Neumann problem, because of (7) and the Neumann boundary condition does not see $\varphi$ where $b \cdot n$ is null).

\section{ASYMPTOTIC ANALYSIS OF THE ERGODIC MEASURE}

1. Proof of Theorem 1. In this subsection, we prove Theorem 1. We do not repeat the argument of $[4,5]$ which states the existence of a positive solution $v_{\dot{\varepsilon}}$ of $(6)-\left(6^{\prime}\right)$ in $W^{1, p}(\Omega) \cap C(\bar{\Omega})$ such that $\max v_{\varepsilon}=1$. We now prove (11). We use the Harnack Inequality (Gilbarg and Trudinger [15, p. 199]), this kind of argument has already been used by Bardi [1]. It shows that, for any $\Omega_{\alpha}, \bar{\Omega}_{\alpha} \subset \Omega$, one has

$$
\operatorname{Inf}_{\Omega_{\alpha}} v_{\varepsilon} \geq \exp (-C / \varepsilon) \sup _{\Omega_{\alpha}} v_{\varepsilon},
$$

where $C$ only depends on $\|b\|_{\infty}$ and $\alpha$. In order to conclude, we have to examine what happens near $\partial \Omega$. Our remark is that the Harnack inequality still holds on $\partial \Omega$ because of the special boundary condition $\left(6^{\prime}\right)$. The proof is exactly the same as for interior points and we only sketch the main steps. We refer the reader to [15] for a complete and general proof.

Let $y$ belong to $\partial \Omega$ and let us choose $R$ small enough such that on

$$
D_{4 R}=B_{4 R}(y) \cap \Omega,
$$

the Sobolev injection of $H_{\Gamma}^{1}\left(D_{4 R}\right)$ into $L^{2 N /(N-2)}\left(D_{4 R}\right)$ holds (we consider the case $N>2$ to shorten the proof), where

$$
H_{\Gamma}^{1}\left(D_{r}\right)=\left\{u \in H^{1}\left(D_{r}\right), u=0 \text { on } \partial D_{r} \backslash \partial \Omega\right\},
$$

i.e. for some constant $C$

$$
\forall v \in H_{\Gamma}^{1}\left(D_{4 R}\right) \quad\|v\|_{2 N /(N-2)} \leq C\|\nabla v\|_{2},
$$

with obvious notations for the norms. This is possible since $D_{4 R}$ is diffeomorphic to a half ball for $R$ small. 
For $\beta \neq 0$ and nonnegative $\eta \in C^{1}(\bar{\Omega})$, we may choose $\eta^{2} v^{\beta}$ (we drop the index $\varepsilon$ ) as a test function in (12). We obtain

$$
\varepsilon \beta \int|\nabla v|^{2} v^{\beta-1} \eta^{2} \leq-2 \varepsilon \int \nabla v v^{\beta} \eta \nabla \eta+\int b v\left(\beta v^{\beta-1} \eta^{2} \nabla v+2 \eta \nabla \eta v^{\beta}\right)
$$

and

$$
\int \eta^{2}|\nabla v|^{2} v^{\beta-1} \leq\left(8 / \beta^{2}\right) \int\left(|\nabla \eta|^{2}+\left(\|b\|_{\infty}^{2} / \varepsilon^{2}\right) \eta^{2}\right) v^{\beta+1}
$$

We set $\gamma=\beta+1$ and

$$
\omega= \begin{cases}v^{(\beta+1) / 2} & \text { if } \beta \neq 1, \\ \log v & \text { if } \beta=-1,\end{cases}
$$

then (15) rewrites (for $\beta \neq-1$ )

$$
\int|\eta \nabla \omega|^{2} \leq\left(32|\gamma| / \beta^{2}\right) \int\left(|\nabla \eta|^{2}+\eta^{2}\|b\|_{\infty}^{2} / \varepsilon^{2}\right) \omega^{2}
$$

Since Sobolev inequality (14) still holds, we may follow [15] and choosing $\eta=0$ in $\Omega \backslash D_{4 R}$,

$$
\|\eta \omega\|_{2 N /(N-2)} \leq(C / \beta)(1+|\gamma|)\left\|\left(|\nabla \eta|+\eta\|b\|_{\infty} / \varepsilon\right) \omega\right\|_{2} .
$$

We may choose also $\eta$ with $\eta \equiv 0$ in $\Omega \backslash D_{r_{2} R}$ and $\eta \equiv 1$ in $D_{r_{1} R}, 1 \leq r_{1}<$ $r_{2} \leq 3$ and $|\nabla \eta| \leq 2 /\left(\left(r_{2}-r_{1}\right) R\right)$, and for $\chi=N /(N-2)$ we obtain (the analogue of formula 8.55 in [15])

$$
\|\omega\|_{L^{2}} \chi_{\left(B_{r_{1}}\right)} \leq C\|\omega\|_{L^{2}\left(B_{r_{2}}\right)}(1+|\gamma|) /\left(r_{2}-r_{1}\right)
$$

with $C_{0}=C\|b\|_{\infty} /(\beta \varepsilon)$.

The end of the proof of the Harnack Inequality is now exactly the same as in [15] and we skip it, moreover the dependence of $C_{0}$ upon $\|b\|_{\infty} / \varepsilon$ is the same as for interior points and thus inequality (11) holds.

To conclude this subsection let us notice that Theorem $8-20$ of [15] is more precise and for $r$ small enough we have

$$
v_{\varepsilon} \geq \sup _{B_{r}} v_{\varepsilon} \exp -\left(C+C r\|b\|_{\infty} / \varepsilon\right)
$$

i.e.

$$
V_{\varepsilon} \leq \inf _{B_{r}} V_{\varepsilon}+C \varepsilon+C\|b\|_{\infty} r
$$

this will be used later.

2. Proof of Theorem 2. We divide the proof of Theorem 2 into three steps. The first one identifies the limit of $V_{\varepsilon}$ as sub- or supersolution of $(5)-\left(5^{\prime}\right)$. Then comparison lemmas for Hamilton-Jacobi equations with discontinuous solutions allow us to conclude. Let us point out that this method simplifies the general program devised by Evans and Ishii [9] to treat large deviation problems. Indeed, we do not prove Lipschitz estimates (which hold if the field 
$b(x)$ is smooth); our proof is reduced to a uniqueness result which however is necessary.

It is easy to see that $V_{\varepsilon}$ is a viscosity solution of (9) in the sense of Lions [21]. But in order to avoid technical difficulties let us consider for any $\varepsilon>0$ some smooth $b_{\varepsilon}$ satisfying (H1) uniformly. Then Theorem 1 still holds for $w_{\varepsilon}$ a solution of

$$
\left\{\begin{array}{l}
-\frac{\varepsilon}{2} \Delta w_{\varepsilon}+\operatorname{div}\left(b_{\varepsilon} w_{\varepsilon}\right)=0, \quad \max _{\bar{\Omega}} w_{\varepsilon}=1, \\
\frac{\varepsilon}{2} \partial w_{\varepsilon} / \partial n-b_{\varepsilon} \cdot n w_{\varepsilon}=0 \text { on } \partial \Omega .
\end{array}\right.
$$

Finally, we may choose $b_{\varepsilon}$ smooth enough so that (19) holds in a classical sense and so that, setting $w_{\varepsilon}=e^{-W_{\varepsilon} / \varepsilon}$, then

$$
V_{\varepsilon}-W_{\varepsilon} \underset{\varepsilon \rightarrow 0}{\longrightarrow} 0 \text { in } C(\bar{\Omega}), \quad b_{\varepsilon}-b \underset{\varepsilon \rightarrow 0}{\longrightarrow} 0 \quad \text { in } C\left(\bar{\Omega} \backslash B_{\delta}\right), \forall \delta>0 .
$$

Now, $W_{\varepsilon}$ satisfies in a classical sense

$$
\begin{aligned}
& -\frac{\varepsilon}{2} \Delta W_{\varepsilon}+\left|\nabla W_{\varepsilon}\right|^{2} / 2+b_{\varepsilon} \cdot \nabla W_{\varepsilon}=\varepsilon \operatorname{div} b_{\varepsilon} \text { in } \Omega, \\
& \partial W_{\varepsilon} / \partial n=-2 b_{\varepsilon} \cdot n \text { on } \partial \Omega,
\end{aligned}
$$

and it is enough to prove Theorem 2 for $W_{\varepsilon}$ rather than $V_{\varepsilon}$. Let us consider the functions defined for any subsequence $\varepsilon_{n} \underset{n \rightarrow \infty}{\longrightarrow} 0$ by

$$
\begin{gathered}
\bar{V}(x)=\limsup _{\varepsilon \rightarrow 0, y \rightarrow x} W_{\varepsilon}(y), \\
\underline{V}(x)=\limsup _{\varepsilon^{\prime} \rightarrow 0, y \rightarrow x} W_{\varepsilon^{\prime}}(y),
\end{gathered}
$$

where $\varepsilon_{n}^{\prime}$ denotes a subsequence extracted of $\varepsilon_{n}$ such that

$$
\bar{V}(0)=\lim _{n \rightarrow \infty} W_{\varepsilon^{\prime}}\left(y_{n}\right) \text { for some } y_{n} \rightarrow 0 .
$$

Let us state our first lemma.

Lemma 1. Let $b \in C(\bar{\Omega} \backslash\{0\})$. Then $\bar{V}(0)=\underline{V}(0)$ and $\bar{V}$ is an upper semicontinuous (u.s.c.) viscosity subsolution of

$$
\begin{gathered}
H(x, \nabla \bar{V}) \leq 0 \quad \text { in } \Omega \backslash\{0\}, \\
\operatorname{Min}(\partial \bar{V} / \partial n+2 b \cdot n, H(x, \nabla \bar{V})) \leq 0 \quad \text { on } \partial \Omega .
\end{gathered}
$$

$\underline{V}$ is a lower semicontinuous (l.s.c.) viscosity supersolution of

$$
\begin{gathered}
H(x, \nabla \underline{V}) \geq 0 \quad \text { in } \Omega \backslash\{0\}, \\
\operatorname{Max}(\partial \underline{V} / \partial n+2 b \cdot n, H(x, \nabla \underline{V})) \geq 0 \quad \text { on } \partial \Omega .
\end{gathered}
$$

Again, we refer the reader to Appendix 1 and to the papers $[2,3,17]$ for discontinuous viscosity solutions of H.J. equations. The meaning of $\left(24^{\prime}\right)$, for example, is that for any $\Phi \in C^{2}(\bar{\Omega})$ and

$$
\operatorname{Max}_{\bar{\Omega}}(\bar{V}-\Phi)=(\bar{V}-\Phi)\left(x_{0}\right), \quad x_{0} \in \partial \Omega
$$


then

$$
\operatorname{Min}\left(H\left(x_{0}, \nabla \Phi\left(x_{0}\right)\right), \partial \Phi / \partial n\left(x_{0}\right)+2 b \cdot n\left(x_{0}\right)\right) \leq 0 .
$$

The proof of Lemma 1 is simple. $\underline{V}(0)=\bar{V}(0)$ is a simple consequence of (18). Let us prove, following [2], that $\bar{V}$ is a subsolution on the boundary. Consider $x_{0} \in \partial \Omega$, such that

$$
\operatorname{Max}_{\Omega}(\bar{V}-\Phi)=(\bar{V}-\Phi)\left(x_{0}\right)>(\bar{V}-\Phi)(x), \quad x \neq x_{0}
$$

(this last inequality is not restrictive). Define $x_{\varepsilon} \in \bar{\Omega}$ by

$$
\underset{\bar{\Omega}}{\operatorname{Max}}\left(W_{\varepsilon}-\Phi\right)=\left(W_{\varepsilon}-\Phi\right)\left(x_{\varepsilon}\right) \text {. }
$$

Then, following [2], $x_{\varepsilon} \rightarrow x_{0}, W_{\varepsilon}\left(x_{\varepsilon}\right) \rightarrow \bar{V}\left(x_{0}\right)$ as $\varepsilon \rightarrow 0$. If $x_{\varepsilon} \in \partial \Omega$, we have

$$
\partial \Phi\left(x_{\varepsilon}\right) / \partial n \leq \partial W_{\varepsilon}\left(x_{\varepsilon}\right) / \partial n \leq 2 b \cdot n,
$$

and if $x_{\varepsilon} \in \partial \Omega$, we have $D^{2}\left(W_{\varepsilon}-\Phi\right)\left(x_{\varepsilon}\right) \leq 0$, therefore

$$
H\left(x_{\varepsilon}, \nabla \Phi\left(x_{\varepsilon}\right)\right) \leq 0 \text {. }
$$

In both cases, we may pass to the limit to get $\left(24^{\prime}\right)$.

In order to compare $\bar{V}$ and $\underline{V}$, we have to build a strict subsolution following the proof of [16]. This is the aim of

Lemma 2. Under assumptions $(\mathrm{H} 1)$ and $(\mathrm{H} 2)$, the u.s.c. function $U=(1-\theta) \bar{V}+$ $\theta \psi_{\delta}$ satisfies, for any $\delta$ small enough,

$$
H(x, \nabla U) \leq-\theta \rho(\delta) \quad \text { in } \Omega \backslash \bar{B}_{\delta},
$$

Proof of Lemma 2. Consider, for any $\Phi \in C^{2}(\bar{\Omega})$,

$$
m=\operatorname{Max}_{\bar{\Omega}}(U-\Phi)=\operatorname{Max}_{\bar{\Omega}}\left(\theta \psi_{\delta}+(1-\theta) \bar{V}-\Phi\right)=(U-\Phi)\left(x_{0}\right) .
$$

We only consider the most difficult case, i.e. $x_{0} \in \partial \Omega$. Then we have to prove that

$$
\operatorname{Min}\left(H\left(x_{0}, \nabla \Phi\left(x_{0}\right)\right)+\theta \rho(\delta), \partial \Phi / \partial n\left(x_{0}\right)+2 b \cdot n\left(x_{0}\right)\right) \leq 0 .
$$

Define $y_{\varepsilon}, x_{\varepsilon} \in \bar{\Omega}$ by

$$
\begin{aligned}
m_{\varepsilon} & =\operatorname{Max}_{x, y \in \bar{\Omega}}\left(\theta \psi_{\delta}(y)+(1-\theta) \bar{V}(x)-|x-y|^{2} /(2 \varepsilon)-\theta \Phi(y)-(1-\theta) \Phi(x)\right) \\
& =\theta \psi_{\delta}\left(y_{\varepsilon}\right)+(1-\theta) \bar{V}\left(x_{\varepsilon}\right)-\left|x_{\varepsilon}-y_{\varepsilon}\right|^{2} /(2 \varepsilon)+\theta \Phi\left(y_{\varepsilon}\right)-(1-\theta) \Phi\left(x_{\varepsilon}\right) .
\end{aligned}
$$

As usual, it is easy to see that (assuming that $x_{0}$ is a strict maximum in (27)) $m_{\varepsilon} \rightarrow m, x_{\varepsilon}, y_{\varepsilon} \rightarrow x_{0},\left|x_{\varepsilon}-y_{\varepsilon}\right|^{2} /(2 \varepsilon) \rightarrow 0$ as $\varepsilon \rightarrow 0$. Several cases may occur:

(i) $x_{\varepsilon} \in \Omega, y_{\varepsilon} \in \Omega$. Then

$$
\begin{gathered}
H\left(x_{\varepsilon},\left(x_{\varepsilon}-y_{\varepsilon}\right) /(1-\theta) \varepsilon+\nabla \Phi\left(x_{\varepsilon}\right)\right) \leq 0, \\
H\left(y_{\varepsilon},\left(x_{\varepsilon}-y_{\varepsilon}\right) / \theta \varepsilon+\nabla \Phi\left(y_{\varepsilon}\right)\right) \leq-\rho(\delta) ;
\end{gathered}
$$


this implies that $\left(x_{\varepsilon}-y_{\varepsilon}\right) / \varepsilon$ remains bounded (since $H(x, p) \rightarrow \infty$ as $|p| \rightarrow \infty$, uniformly). Therefore

$$
H\left(x_{\varepsilon},-\left(x_{\varepsilon}-y_{\varepsilon}\right) / \theta \varepsilon+\nabla \Phi\left(y_{\varepsilon}\right)\right) \leq-\rho(\delta)+C\left|x_{\varepsilon}-y_{\varepsilon}\right| .
$$

Since $H$ is convex in $p$, we get

$$
H\left(x_{\varepsilon},(1-\theta) \nabla \Phi\left(x_{\varepsilon}\right)+\theta \nabla \Phi\left(y_{\varepsilon}\right)\right) \leq-\rho(\delta)+C\left|x_{\varepsilon}-y_{\varepsilon}\right|,
$$

and we conclude setting $\varepsilon \rightarrow 0$ if the case (i) occurs for a subsequence $\varepsilon_{n} \rightarrow 0$.

(ii) $y_{\varepsilon} \in \partial \Omega$. Then, from (H2) we obtain

$$
-\left(x_{\varepsilon}-y_{\varepsilon}\right) \cdot n / \theta \varepsilon+\partial \Phi / \partial n\left(y_{\varepsilon}\right)+2 b \cdot n \leq 0 \text {. }
$$

Since $y_{\varepsilon} \in \partial \Omega$, we have (see Lions [22] and Perthame and Sanders [24])

$$
\left(x_{\varepsilon}-y_{\varepsilon}\right) \cdot n \leq C\left|x_{\varepsilon}-y_{\varepsilon}\right|^{2} \text {. }
$$

Therefore

$$
\partial \Phi / \partial n\left(y_{\varepsilon}\right)+2 b \cdot n \leq C\left|x_{\varepsilon}-y_{\varepsilon}\right|^{2} / \varepsilon \rightarrow 0 \text { as } \varepsilon \rightarrow 0,
$$

and we conclude again in this case.

(iii) $y_{\varepsilon} \in \Omega, x_{\varepsilon} \in \partial \Omega$ and $H\left(x_{\varepsilon},\left(x_{\varepsilon}-y_{\varepsilon}\right) /(1-\theta) \varepsilon+\nabla \Phi\left(x_{\varepsilon}\right)\right) \leq 0$. This case is analogous to case (i).

(iv) $y_{\varepsilon} \in \Omega, x_{\varepsilon} \in \partial \Omega$ and $\left(x_{\varepsilon}-y_{\varepsilon}\right) \cdot n /(1-\theta) \varepsilon+\partial \Phi / \partial n\left(x_{\varepsilon}\right)+2 b \cdot n\left(x_{\varepsilon}\right) \leq 0$. This case is analogous to case (ii).

Thus Lemma 2 is proved.

Our last and crucial result is

Lemma 3. Under assumptions ( $\mathrm{H} 1)$ and $(\mathrm{H} 2), \underline{V} \geq \bar{V}$ in $\bar{\Omega}$.

Proof of Lemma 3. We are going to prove that for any $\theta$ and $\delta, U \leq \underline{V}+C \rho^{\prime}(\delta)$ for some modulus of continuity $\rho^{\prime}$. Consider $x_{0} \in \bar{\Omega} \backslash B_{\delta}$ such that

$$
m=\underset{\bar{\Omega} \backslash B_{\delta}}{\operatorname{Max}}(U-\underline{V})=(U-\underline{V})\left(x_{0}\right) .
$$

If $x_{0} \in \partial B_{\delta}$, then the inequality is clear since $\bar{V}(0)=\underline{V}(0)$. If $x_{0} \in \Omega \backslash \bar{B}_{\delta}$, we may conclude as usual and thus we only treat the case $x_{0} \in \partial \Omega$. Let us fix $\alpha>0$ and set

$$
\begin{aligned}
m_{\varepsilon}^{\alpha} & =\operatorname{Max}_{x, y \in \bar{\Omega}}\left\{U(y)-\underline{V}(x)-2 b_{\varepsilon} \cdot(x-y)-\left|x_{\varepsilon}-y_{\varepsilon}\right|^{2} / 2 \varepsilon+\alpha(\xi(x)+\xi(y))\right\} \\
& \left.=U\left(x_{\varepsilon}\right)-\underline{V}\left(x_{\varepsilon}\right)-2 b_{\varepsilon} \cdot\left(x_{\varepsilon}-y_{\varepsilon}\right)-\left|x_{\varepsilon}-y_{\varepsilon}\right|^{2} / 2 \varepsilon+\alpha\left(\xi\left(x_{\varepsilon}\right)+\xi\left(y_{\varepsilon}\right)\right)\right\},
\end{aligned}
$$

where $\xi \in C^{1}(\bar{\Omega}), \xi=0$ on $\partial \Omega, \nabla \xi \cdot n=1$ on $\partial \Omega, b_{\varepsilon} \equiv b_{\varepsilon}(x)$ satisfies $b_{\varepsilon} \rightarrow b$ uniformly as $\varepsilon \rightarrow 0$, and $b_{\varepsilon}$ is $C^{1}$ with $\left|\nabla b_{\varepsilon}\right| \sqrt{\varepsilon} \rightarrow 0$ as $\varepsilon \rightarrow 0$. We set

$$
m^{\alpha}=\operatorname{Max}_{x \in \bar{\Omega}}\{U(x)-\underline{V}(x)+2 \alpha \xi(x)\} .
$$


We see that $m^{\alpha} \leq m_{\varepsilon}^{\alpha}$, and we may extract a subsequence of $\varepsilon$ such that

$$
x_{\varepsilon} \rightarrow x^{\alpha}, \quad y_{\varepsilon} \rightarrow x^{\alpha} \quad \text { as } \varepsilon \rightarrow 0,
$$

because $\left|x_{\varepsilon}-y_{\varepsilon}\right|^{2} / 2 \varepsilon$ remains bounded. From (27), we obtain

$$
\begin{aligned}
m^{\alpha} \leq & m_{\varepsilon}^{\alpha}=U\left(y_{\varepsilon}\right)-U\left(x^{\alpha}\right)-\underline{V}\left(x_{\varepsilon}\right)+\underline{V}\left(x^{\alpha}\right)+U\left(x^{\alpha}\right)-\underline{V}\left(x^{\alpha}\right) \\
& +\alpha\left(\xi\left(x_{\varepsilon}\right)+\xi\left(y_{\varepsilon}\right)\right)+2 b_{\varepsilon}\left(x_{\varepsilon}-y_{\varepsilon}\right)-\left|x_{\varepsilon}-y_{\varepsilon}\right|^{2} / 2 \varepsilon \\
& \leq m^{\alpha}-\left|x_{\varepsilon}-y_{\varepsilon}\right|^{2} / 2 \varepsilon+\rho\left(\left|x_{\varepsilon}-x^{\alpha}\right|+\left|y_{\varepsilon}-x^{\alpha}\right|\right),
\end{aligned}
$$

where $\rho$ denotes a modulus of continuity (depending on the point $x^{\alpha}$ ).

This shows that $\left|x_{\varepsilon}-y_{\varepsilon}\right|^{2} / 2 \varepsilon \rightarrow 0$ as $\varepsilon \rightarrow 0$. Now, we set

$$
\begin{aligned}
& p=-\left(x_{\varepsilon}-y_{\varepsilon}\right) / \varepsilon-\alpha \nabla \xi\left(x_{\varepsilon}\right)-2 b_{\varepsilon}\left(x_{\varepsilon}\right)-2 \nabla b_{\varepsilon}\left(x_{\varepsilon}\right)\left(x_{\varepsilon}-y_{\varepsilon}\right), \\
& q=-\left(x_{\varepsilon}-y_{\varepsilon}\right) / \varepsilon+\alpha \nabla \xi\left(y_{\varepsilon}\right)-2 b_{\varepsilon}\left(x_{\varepsilon}\right) .
\end{aligned}
$$

As in the proof of Lemma 2, we obtain if $y_{\varepsilon} \in \partial \Omega$

$$
q \cdot n \geq-C\left|x_{\varepsilon}-y_{\varepsilon}\right|^{2} / \varepsilon+\alpha-2 b_{\varepsilon}\left(x_{\varepsilon}\right) \cdot n,
$$

and if $x_{\varepsilon} \in \partial \Omega$

$$
p \cdot n \leq C\left|x_{\varepsilon}-y_{\varepsilon}\right|^{2} / \varepsilon-\alpha-2 b_{\varepsilon}\left(x_{\varepsilon}\right) \cdot n+C\left|\nabla b_{\varepsilon}\right| \cdot \sqrt{\varepsilon} .
$$

For $\varepsilon$ small enough, this reduces to

$$
p \cdot n \leq-2 b\left(x_{\varepsilon}\right) \cdot n-\alpha / 2, \quad q \cdot n \geq-2 b\left(y_{\varepsilon}\right) \cdot n+\alpha / 2 .
$$

Thus, the viscosity characterization of $U$ and $\underline{V}$ gives always (for $\varepsilon$ small enough)

$$
H\left(x_{\varepsilon}, p\right) \geq 0, \quad H\left(y_{\varepsilon}, q\right) \leq-\theta \rho(\delta),
$$

thus $q$ remains uniformly bounded and the second inequality reads

$$
H\left(x_{\varepsilon}, q\right) \leq-\theta \rho(\delta)+C\left|b\left(x_{\varepsilon}\right)-b\left(y_{\varepsilon}\right)\right| \leq-\theta \rho(\delta) / 2
$$

for $\varepsilon$ small enough. This gives a contradiction when $\alpha$ tends to 0 .

This shows that $x_{0} \in \Omega \backslash B_{\delta}$ and concludes the proof of Lemma 3 setting $\theta$ and $\delta$ to zero.

Remark. This proof is very close to the proof of uniqueness of viscosity solutions for H.J. Equations with Neumann boundary conditions of [22, 24]; it can be extended to discontinuous solutions with no change. This particularly simple proof that we have presented here has been taken from Ishii and Lions [18].

The proof of Theorem 2 is now nearly complete. Since $\bar{V}$ is obviously greater than $\underline{V}$, we have proved that $\underline{V}=\bar{V}$. This means that $W_{\varepsilon}$ converges uniformly to some $V_{0}$, the unique solution of $(24)-\left(24^{\prime}\right),(25)-\left(25^{\prime}\right)$. It remains to notice that this problem is equivalent to the state constraints problem $(5)-\left(5^{\prime}\right)$. This is clear from the following formula proved in [22]. If $V_{0}$ satisfies $(25)-\left(25^{\prime}\right)$, then for any $\phi \in C^{1}(\bar{\Omega})$ and any $x_{0} \in \partial \Omega, \exists \theta \in[0,1]$ such that

$$
H\left(x_{0}, \nabla \phi\left(x_{0}\right)-\theta\left(\partial \phi / \partial n\left(x_{0}\right)+2 b \cdot n\right)^{+} n\right) \geq 0,
$$


i.e.

$$
\begin{aligned}
H\left(x_{0}, \nabla \phi\left(x_{0}\right)\right)+\theta(\partial \phi / \partial n+2 b \cdot n)^{+} \\
\cdot\left\{\theta(\partial \phi / \partial n+2 b \cdot n)^{+} / 2-(2 b \cdot n+\partial \phi / \partial n)+b \cdot n\right\} \geq 0 .
\end{aligned}
$$

Since $b \cdot n \leq 0$, this implies that

$$
H\left(x_{0}, \nabla \phi\left(x_{0}\right)\right) \geq 0
$$

and we have recovered the boundary condition $\left(5^{\prime}\right)$. By uniqueness (we do not prove the uniqueness for the boundary condition $\left(5^{\prime}\right)$ which a simple consequence of [25]) the two problems $(5)-\left(5^{\prime}\right)$ and $(24)-\left(24^{\prime}\right),(25)-\left(25^{\prime}\right)$ are therefore equivalent, and their solution is given by

$$
\begin{array}{r}
Q(x)=\operatorname{Inf}\left\{\int_{0}^{\tau} L(y(s), \dot{y}(s)) d s ; y \in H^{1}\left(\mathbf{R}^{+}\right), y(0)=x,\right. \\
y(\tau)=0, y(s) \in \bar{\Omega} \backslash\{0\} \text { for } 0 \leq s<\tau\}
\end{array}
$$

(this formula is clearly equivalent to (3) if $b$ is continuous). Since $\operatorname{Min}_{\bar{\Omega}} V_{0}=0$ we get $V_{0}=Q$, thus Theorem 2 is proved.

\section{ASYMPTOTIC ANALYSIS OF $u_{\varepsilon}$ AND $z_{\varepsilon}$}

1. Proof of Theorem 4. We begin by proving Theorem 4, its proof is simpler because it is not necessary to perform a precise boundary layer analysis. Let us first point out that $z_{\varepsilon}$ exists because $\mu_{\varepsilon}$ is chosen so that $\int_{\partial \Omega} v_{\varepsilon} b \cdot n\left(\varphi-\mu_{\varepsilon}\right)=0$, which is the solvability condition for

$$
\left\{\begin{array}{l}
L_{\varepsilon} z_{\varepsilon}=-\frac{\varepsilon}{2} \Delta z_{\varepsilon}-b \cdot \nabla z_{\varepsilon}=0, \quad z_{\varepsilon} \in H^{1}(\Omega) \cap L^{\infty}(\Omega), \\
\frac{\varepsilon}{2} \partial z_{\varepsilon} / \partial n=-b \cdot n\left(\varphi-\mu_{\varepsilon}\right) \quad \text { on } \partial \Omega, \quad \int_{\Omega} v_{\varepsilon} z_{\varepsilon} d x=0
\end{array}\right.
$$

(see $[4,5])$. To study (29) let us begin by some preliminary lemmas.

Let $\delta$ be a positive number as small as necessary and let $O_{\delta}$ be a smooth open subset of $\Omega$ containing 0 such that $d\left(\partial \Omega, O_{\delta}\right)>\delta$. Let $m:=m\left(\varepsilon, O_{\delta}\right)$ satisfy

$$
\left\{\begin{array}{l}
L_{\varepsilon} m=-\frac{\varepsilon}{2} \Delta m+\operatorname{div}(b m)=0, \quad m \in H^{1}\left(\Omega \backslash \bar{O}_{\delta}\right) \cap C\left(\bar{\Omega} \backslash O_{\delta}\right), \\
\frac{\varepsilon}{2} \partial m / \partial n-b \cdot n m=0 \quad \text { on } \partial \Omega, \quad m=1 \text { on } \partial O_{\delta} .
\end{array}\right.
$$

Because of the Dirichlet boundary condition, there exists a unique solution to (30) which satisfies the maximum principle and thus

$$
v_{\varepsilon} \leq m \leq 1 \text { on } \bar{\Omega} \backslash O_{\delta} .
$$

We are going to follow the same asymptotic analysis for $m$ as for $v_{\varepsilon}$, therefore we set

$$
\begin{gathered}
m=e^{-M_{\varepsilon} / \varepsilon}, \quad 0 \leq M_{\varepsilon} \leq V_{\varepsilon}, \\
-\frac{\varepsilon}{2} \Delta M_{\varepsilon}+\left|\nabla M_{\varepsilon}\right|^{2} / 2+\left(b, \nabla M_{\varepsilon}\right)=\varepsilon \operatorname{div} b \quad \text { in } \Omega \backslash \bar{O}_{\delta}, \\
\partial M_{\varepsilon} / \partial n=-2 b \cdot n \quad \text { on } \partial \Omega, \quad M_{\varepsilon}=0 \quad \text { on } \partial O_{\delta} .
\end{gathered}
$$


Lemma 4. Under assumptions $(\mathrm{H} 1)$ and $(\mathrm{H} 2), M_{\varepsilon}$ converges uniformly on $\Omega \backslash O_{\delta}$ to the unique solution $M \in C\left(\bar{\Omega} \backslash O_{\delta}\right)$ of

$$
\begin{gathered}
H(x, \nabla M)=0 \quad \text { in } \Omega \backslash \bar{O}_{\delta}, \\
M=0 \quad \text { on } \partial O_{\delta}, \\
H(x, \nabla M) \geq 0 \quad \text { on } \partial \Omega .
\end{gathered}
$$

Proof of Theorem 4. Define $\bar{M}, \underline{M}$ as in the proof of Lemma 2; these functions are sub- and supersolutions of (33). We claim that $\bar{M}$ satisfies $\left(33^{\prime}\right)$. Indeed, one can easily check that for $\lambda$ large enough the function $d(x)=\lambda d\left(x, \partial O_{\delta}\right)$ is a strict supersolution of (32) in a small neighborhood $V$ of $\partial O_{\delta}$ (we assume that $d$ is smooth in $V$ ). For $\lambda$ large enough, $\lambda d$ is larger than $V_{\varepsilon}$ on $\partial V$. This proves that $M_{\varepsilon}$ is smaller than $\lambda d$, and thus $\left(33^{\prime}\right)$ holds for $\bar{M}, \underline{M}$. Then, we choose $\delta^{\prime}$ small enough so that $B_{\delta^{\prime}} \subset O_{\delta}$. For $\beta$ small enough, the function (see (H2)) $\beta \psi_{\delta^{\prime}}$ is still a strict subsolution of (33) and satisfies

$$
\partial \beta \psi_{\delta^{\prime}} / \partial n \leq-2 b \cdot n \quad \text { on } \partial \Omega, \quad \beta \psi_{\delta^{\prime}} \rightarrow 0 \quad \text { as } \beta \rightarrow 0 \text { on } \partial O_{\delta} .
$$

Therefore, we may apply the proof of Theorem 2 without any modification and we obtain that $\bar{M}=\underline{M}$, and that the Neumann condition for (33) is equivalent to $\left(33^{\prime \prime}\right)$.

Lemma 5. With the notations of Lemma 4, there exists a constant $l(\delta)>0$ such that for any choice of the smooth open subset of $\Omega O_{\delta}$ containing 0 and satisfying $d\left(\partial \Omega, \partial O_{\delta}\right) \geq \delta>0$, then $M_{\varepsilon} \geq 3 l(\delta)$ on $\partial \Omega$, for $0<\varepsilon<1$.

Proof of Lemma 5. Applying the maximum principle to (32) the worst constant $l$ is obtained when

$$
O_{\delta}=\{x ; d(x, \partial \Omega)>\delta\} .
$$

In this case, assume that $\operatorname{Min}_{\partial \Omega}=0=M\left(x_{0}\right), x_{0} \in \partial \Omega$. We will obtain a contradiction by using the maximum principle in (33). It holds because we have a strict subsolution. Indeed, consider

$$
\begin{aligned}
\operatorname{Min}_{x, y \in \bar{\Omega}}\left\{M(x)-\beta \psi_{\delta^{\prime}}(y)\right. & +C\left|x-x_{0}\right|^{2}-2 b_{\varepsilon}(x) \cdot(x-y) \\
& \left.+C|x-y|^{2} /(2 \varepsilon)+\beta(\xi(x)+\xi(y))\right\}
\end{aligned}
$$

(we use the notation of the proofs of Lemmas 2, 3, 4); it is attained at points $x_{\varepsilon}, y_{\varepsilon}$. As usual, we have, since $M$ is Lipschitz continuous,

$$
\left|x_{\varepsilon}-y_{\varepsilon}\right| \leq C \varepsilon, \quad x_{\varepsilon}, y_{\varepsilon} \rightarrow x_{\beta} \quad \text { as } \varepsilon \rightarrow 0, \quad x_{\beta} \rightarrow x_{0} \quad \text { as } \beta \rightarrow 0 .
$$

We obtain a contradiction as in Lemma 2. For example, if $x_{\varepsilon}, y_{\varepsilon} \in \Omega$, then

$$
\begin{gathered}
H\left(x_{\varepsilon},-2 C\left(x_{\varepsilon}-x_{0}\right)-2 C\left(x_{\varepsilon}-y_{\varepsilon}\right) / \varepsilon+\beta \nabla \xi\left(x_{\varepsilon}\right)+2 b\left(x_{\varepsilon}\right)+2 x_{\varepsilon} \cdot \nabla b_{\varepsilon}\right) \geq 0, \\
H\left(y_{\varepsilon},-2 C\left(x_{\varepsilon}-y_{\varepsilon}\right) / \varepsilon+\beta \nabla \xi\left(y_{\varepsilon}\right)+2 b\left(x_{\varepsilon}\right)\right) \leq-\beta \rho\left(\delta^{\prime}\right),
\end{gathered}
$$

and this is a contradiction setting $\varepsilon$ and $\beta$ to 0 . The other cases are also mere adaptations and we skip them. 
We may now turn to the proof of Theorem 4. We divide its proof into two steps; first, we show the exponential decay of $z_{\varepsilon}$ in compact subsets of $\Omega$, then we conclude by an analysis of the boundary layer. We will prove an estimate on the decay and the $L^{\infty}$ norm of $z_{\varepsilon}$ which only depends on $\|b\|_{\infty}, \sup _{\partial \Omega}(b \cdot n)$, and $\|\varphi\|_{\infty}$; therefore it is enough to assume that $\varphi$ and $b$ are smooth.

Step 1. Decay in compact subsets. Let a positive number $\gamma$ be given and let us prove an estimate on the decay of $z_{\varepsilon}$ in $\{x ; d(\partial \Omega, x)>\gamma\}$.

First, we choose $m=v_{\varepsilon}$ (formally, $\delta=0$ in Lemmas 4 and 5) and we multiply (29) by $z_{\varepsilon} m$. Green's formula gives

$$
\frac{\varepsilon}{2} \int_{\Omega}\left|\nabla z_{\varepsilon}\right|^{2} m=\int_{\partial \Omega} m z_{\varepsilon} b \cdot n\left(\varphi-\mu_{\varepsilon}\right) \leq C\left\|z_{\varepsilon}\right\|_{\infty} e^{-3 l(\gamma) / \varepsilon}
$$

because the result of Lemma 5 applies for $\delta=0$ and $m=v_{\varepsilon}$. Since $\left|\nabla V_{0}\right| \leq$ $2\|b\|_{\infty}$, on the ball

$$
B_{1}=\left\{x ;|x| \leq l(\gamma) /\left(3\|b\|_{\infty}\right)\right\}
$$

we have (choose $\varepsilon$ small enough) $m \geq e^{-l(\gamma) / \varepsilon}$ (from the definition of $l(\gamma)$ we may assume that $l(\gamma) \leq 2 \gamma\|b\|_{\infty}$, so that $\left.B_{1} \subset \Omega\right)$. Therefore

$$
\int_{B_{1}}\left|\nabla z_{\varepsilon}\right|^{2} \leq C\left\|z_{\varepsilon}\right\|_{\infty}^{-2 l(\gamma) / \varepsilon} / \varepsilon
$$

This proves that (denoting $f_{C_{1}} f$ the average of $f$ on $C$ )

$$
\left\|z_{\varepsilon}-f_{B_{1}} z_{\varepsilon}\right\|_{L^{2}\left(B_{1}\right)}^{2} \leq C\left\|z_{\varepsilon}\right\|_{\infty} e^{-2 l(\gamma) / \varepsilon} / \varepsilon,
$$

and that

$$
\left\|\Delta z_{\varepsilon}\right\|_{L^{2}\left(B_{1}\right)}^{2} \leq C\left\|z_{\varepsilon}\right\|_{\infty} e^{-2 l(\gamma) / \varepsilon} / \varepsilon^{3},
$$

by local regularity results for the Laplace operator. These last two inequalities give

i.e. by Sobolev injections:

$$
\left\|z_{\varepsilon}-f_{B} z_{\varepsilon}\right\|_{H^{2}\left(B_{1} / 2\right)}^{2} \leq C\left\|z_{\varepsilon}\right\|_{\infty} e^{-2 l(\gamma) / \varepsilon} / \varepsilon^{3},
$$

$$
\varepsilon^{2}\left\|\Delta z_{\varepsilon}\right\|_{L^{2 N /(N-2)}\left(B_{1} / 2\right)}^{2} \leq C\left\|\nabla z_{\varepsilon}\right\|_{L^{2 N /(N-2)}\left(B_{1} / 2\right)}^{2} \leq C\left\|z_{\varepsilon}\right\|_{\infty} e^{-2 l(\gamma) / \varepsilon} / \varepsilon^{3} .
$$

And in a finite number of iterations (say $p$ depending on $N$ ) of this procedure we get, for $C_{1}=\left\{x ;|x| \leq l(\gamma) /\left(2^{p+1}\|b\|_{\infty}\right) ; d(x, \partial \Omega)>\gamma\right\}$,

$$
\left\|z_{\varepsilon}-f_{C_{1}} z_{\varepsilon}\right\|_{L^{\infty}\left(C_{1}\right)}^{2} \leq C\left\|z_{\varepsilon}\right\|_{\infty} e^{-l(\gamma) / \varepsilon},
$$

i.e.

$$
\left\|z_{\varepsilon}-z_{\varepsilon}(0)\right\|_{L^{\infty}\left(C_{1}\right)}^{2} \leq C\left\|z_{\varepsilon}\right\|_{\infty} e^{-l(\gamma) / \varepsilon} .
$$


We may now iterate this argument. Consider $z^{(1)}$ solution of

$$
\begin{aligned}
& L_{\varepsilon} z^{(1)}=-\frac{\varepsilon}{2} \Delta z^{(1)}-b \cdot \nabla z^{(1)}=0, \quad z^{(1)} \in H^{1}\left(\Omega \backslash C_{1}\right) \cap L^{\infty}\left(\Omega \backslash C_{1}\right), \\
& \frac{\varepsilon}{2} \partial z^{(1)} / \partial n=-b \cdot n\left(\varphi-\mu_{\varepsilon}\right) \quad \text { on } \partial \Omega, \quad z^{(1)}=0 \quad \text { on } \partial C_{1} .
\end{aligned}
$$

By the maximum principle one has $\left\|z^{(1)}-z_{\varepsilon}\right\|_{\infty} \leq C\left\|z_{\varepsilon}\right\|_{\infty} e^{-l(\gamma) / \varepsilon}$. We choose now $\delta=\gamma, O_{\delta}=C_{1}$, and we denote by $m$ the solution of (30) for this new choice. Lemma 5 applies and Green's formula gives

$$
\frac{\varepsilon}{2} \int_{\Omega \backslash C_{1}}\left|\nabla z^{(1)}\right|^{2} m=\int_{\partial \Omega} m z^{(1)} b \cdot n\left(\varphi-\mu_{\varepsilon}\right) \leq C\left\|z^{(1)}\right\|_{\infty} e^{-3 l(\gamma) / \varepsilon} .
$$

The same argument as before shows that on the set

$$
B_{2}=\left\{x \in \Omega \backslash \bar{C}_{1} ; d\left(x, \partial C_{1}\right) \leq l(\gamma) /\left(3\|b\|_{\infty}\right)\right\}
$$

we have

$$
\int_{B_{2}}\left|z^{(1)}\right|^{2} \leq C\left\|z^{(1)}\right\|_{\infty} e^{-2 l(\gamma) / \varepsilon} / \varepsilon
$$

i.e. on

$$
C_{2}=\left\{x \in \Omega \backslash C_{1} ; d\left(x, \partial C_{1}\right) \leq l(\gamma) /\left(2^{p+1}\|b\|_{\infty}\right) ; d(x, \partial \Omega)>\gamma\right\},
$$

we obtain

$$
\left\|z^{(1)}-z^{(1)}(0)\right\|_{L^{\infty}\left(C_{2}\right)}^{2} \leq C\left\|z^{(1)}\right\|_{\infty} e^{-l(\gamma) / \varepsilon}
$$

i.e.

$$
\left\|z_{\varepsilon}-z_{\varepsilon}(0)\right\|_{L^{\infty}\left(C_{2}\right)}^{2} \leq C\left\|z_{\varepsilon}\right\|_{\infty} e^{-l(\gamma) / \varepsilon} .
$$

Finally, a finite number of iterations of this argument shows that

$$
\left\|z_{\varepsilon}-z_{\varepsilon}(0)\right\|_{L^{\infty}\left(\Omega_{\gamma}\right)}^{2} \leq C\left\|z_{\varepsilon}\right\|_{\infty} e^{-l(\gamma) / \varepsilon}, \quad \Omega_{\gamma}=\{x ; d(x, \partial \Omega)>\gamma\} .
$$

Step 2. Boundary layer analysis. Let us build a super- and a subsolution on the boundary. Consider $d(x)=d(x, \partial \Omega)$ and

$$
f(x)=\mu e^{-\lambda d(x) / \varepsilon} .
$$

We claim that for $\lambda$ small enough and $\mu$ large enough, $f(x)$ is a supersolution of (29) on a neighborhood of $\partial \Omega$. A simple computation shows that

$$
\begin{aligned}
-\frac{\varepsilon}{2} \Delta f-b \cdot \nabla f & =\lambda \mu f\left\{\Delta d-\lambda|\nabla d|^{2} / \varepsilon+2 b \cdot|\nabla d| / \varepsilon\right\} / 2 \\
& \geq \lambda \mu f\{\Delta d-\lambda / \varepsilon-2 b \cdot n / \varepsilon-\rho(d(x) / \varepsilon)\} / 2 \geq 0
\end{aligned}
$$

for $\lambda$ small enough and for some modulus of continuity $\rho$ (we choose $\gamma$ such that $\left.\rho(\gamma) \leq \min _{\partial \Omega}(-b \cdot n)\right)$, and

$$
\varepsilon \partial f / \partial n=\lambda \mu \geq 2|b \cdot n| \cdot\left|\varphi-\mu_{\varepsilon}\right| .
$$

From (34), we see that if we choose $\lambda \leq l(\gamma) /(4 \gamma)$ and

$$
\mu=C+C\left\|z_{\varepsilon}\right\|_{\infty}^{1 / 2} e^{-l(\gamma) /(4 \varepsilon)}
$$


then $f \geq z_{\varepsilon}-z_{\varepsilon}(0)$ on $\partial \Omega_{\gamma}$; thus, we have

$$
\begin{array}{ll}
z_{\varepsilon}-z_{\varepsilon}(0) \leq f \leq C+C\left\|z_{\varepsilon}\right\|_{\infty}^{1 / 2} e^{-l(\gamma) /(4 \varepsilon)} e^{-l(\gamma) d(x) /(4 \gamma \varepsilon)} & \text { on }\{x ; d(x)<\gamma\}, \\
z_{\varepsilon}-z_{\varepsilon}(0) \leq C+C\left\|z_{\varepsilon}\right\|_{\infty}^{1 / 2} e^{-l(\gamma) /(4 \varepsilon)} \quad \text { in } \Omega . &
\end{array}
$$

We obtain the other inequality by changing the sign of $\mu$ and this proves that $z_{\varepsilon}$ is uniformly bounded and (34) gives the exponential decay of $z_{\varepsilon}-z_{\varepsilon}(0)$. By the integral condition on $z_{\varepsilon}$, we see that $z_{\varepsilon}(0)$ is exponentially small and this concludes the proof of Theorem 4.

2. Proof of Theorem 3. The proof of Theorem 3 is more difficult because a boundary layer exists on $u_{\varepsilon}$ and not only on its normal derivative as is the case for $z_{\varepsilon}$. We divide its proof into three steps. First, we make a rough estimate of $\partial u_{\varepsilon} / \partial n$ on $\partial \Omega$. Then we deduce the exponential decay inside $\Omega$. Finally, we perform the boundary layer analysis to prove (7).

First of all, let us notice that it is enough to treat the case where $\varphi \in$ $C^{1, \beta}(\partial \Omega)$, the other cases are deduced by approximating $\varphi$ properly.

Step 1. Estimate on $\partial u_{\varepsilon} / \partial n$. Let $\underline{\varphi} \in C^{2}(\Omega) \cap C^{1, \beta}(\bar{\Omega})$ satisfy

$$
\begin{array}{ll}
-\Delta \underline{\varphi}=0 & \text { in } \Omega, \\
\underline{\varphi}=\varphi & \text { on } \partial \Omega .
\end{array}
$$

Setting $d(x)=d(x, \partial \Omega)$ for $d(x)<\delta$ small enough the function

$$
h(x)=u_{\varepsilon}-\underline{\varphi} e^{-\lambda d(x) / \varepsilon}
$$

is smooth and satisfies

$$
\begin{array}{r}
-\frac{\varepsilon}{2} \Delta h-b \cdot \nabla h \\
\quad=e^{-\lambda d(x) / \varepsilon}\left\{-\lambda \varepsilon \nabla \underline{\varphi} \cdot \nabla d+\underline{\varphi}\left(\lambda^{2}|\nabla d|^{2}-\lambda \varepsilon \Delta d-2 \lambda b \cdot \nabla d\right)+2 \varepsilon b \cdot \nabla \underline{\varphi}\right\} /(2 \varepsilon), \\
h=0 \quad \text { on } \partial \Omega .
\end{array}
$$

Let us choose $\lambda$ large enough so that, for any $\varepsilon \leq 1$ (and with the same notations as in the proof of Theorem 4)

$$
\lambda|\nabla d|^{2} \geq \varepsilon|\Delta d|+2|b \cdot \nabla d|+1 \text { on } \Omega_{\delta} .
$$

Then we set $g=\mu\left(1-e^{-\lambda d(x) / \varepsilon}\right)$ and we have

$$
\begin{gathered}
\frac{\varepsilon}{2} \Delta g-b \cdot \nabla g=\lambda \mu e^{-\lambda d(x) / \varepsilon}\left\{\lambda|\nabla d|^{2}-\varepsilon \Delta d-b \cdot \nabla d\right\} /(2 \varepsilon) \geq \lambda \mu e^{-\lambda d(x) / \varepsilon} /(2 \varepsilon), \\
g=0 \text { on } \partial \Omega .
\end{gathered}
$$

Therefore, $g$ is a supersolution of (36) for $\mu$ large enough and it satisfies $g \geq \mu / 2 \geq h$ on $\partial \Omega_{\delta}$, hence

$$
g \geq h, \quad \partial h / \partial n \geq-\lambda \mu / \varepsilon \text { on } \partial \Omega .
$$

In the same way (choosing $\mu \leq 0$ ) we obtain that $\varepsilon \partial u_{\varepsilon} / \partial n$ is bounded in $L^{\infty}$. 
Step 2. Exponential decay in subsets of $\Omega$. As before, we multiply (1) by $u_{\varepsilon} \cdot v_{\varepsilon}$ and Green's formula gives

$$
-\frac{\varepsilon}{2} \int_{\Omega}\left|\nabla u_{\varepsilon}\right|^{2} v_{\varepsilon}=\int_{\partial \Omega} \frac{\varepsilon}{2} v_{\varepsilon} \varphi \frac{\partial u_{\varepsilon}}{\partial n} \leq C e^{-3 l(\gamma) / \varepsilon}
$$

(using Step 1 and Lemma 5 with $m=v_{\varepsilon}$ ). Thus, the proof of Theorem 4 applies without any modification and (34) reads (the term of the form $\left\|z_{\varepsilon}\right\|_{\infty}$ has disappeared)

$$
\left\|u_{\varepsilon}-u_{\varepsilon}(0)\right\|_{L^{\infty}\left(\Omega_{\gamma}\right)}^{2} \leq C e^{-l(\gamma) / \varepsilon}, \quad \Omega_{\gamma}=\{x ; d(x, \partial \Omega)>\gamma\},
$$

which proves directly the exponential decay in compact subsets of $\Omega$.

Step 3. Boundary layer analysis. In order to prove formula (7) and to find $u_{\varepsilon}(0)$ let us introduce a parameter $\gamma$ as small as necessary, and $u_{+}$and $u_{-}$solutions of

$$
\begin{aligned}
& -\frac{\varepsilon}{2} \Delta u_{+}-b \cdot \nabla u_{+}=0 \quad \text { in } \Omega \backslash \bar{\Omega}_{\gamma}, \\
& u_{+}=\varphi-u_{\varepsilon}(0)+C_{\infty} \quad \text { on } \partial \Omega, \\
& u_{+}=e^{-r(\gamma) / \varepsilon}+u_{\varepsilon}-u_{\varepsilon}(0) \quad \text { on } \partial \Omega_{\gamma}, \\
& -\frac{\varepsilon}{2} \Delta u_{-}-b \cdot \nabla u_{-}=0 \quad \text { in } \Omega \backslash \bar{\Omega}_{\gamma}, \\
& u_{-}=-\varphi+u_{\varepsilon}(0)+C_{\infty} \quad \text { on } \partial \Omega, \\
& u_{-}=e^{-r(\gamma) / \varepsilon}-u_{\varepsilon}-u_{\varepsilon}(0) \quad \text { on } \partial \Omega_{\gamma},
\end{aligned}
$$

where $r(\gamma)$ will be chosen (small enough, and $r(\gamma) \leq-l(\gamma) / 2$ ) later on, and $C_{\infty}=\left\|\varphi-u_{\varepsilon}(0)\right\|_{\infty}+1$. Notice that $u_{\varepsilon}-u_{\varepsilon}(0)=\left(u_{+}-u_{-}\right) / 2$. Let us determine the behavior of $u_{+}$on $\partial \Omega$. To do this, we set $u_{+}=e^{-U / \varepsilon}$, and we have

$$
\begin{aligned}
& -\frac{\varepsilon}{2} \Delta U+|\nabla U|^{2} / 2-b \cdot \nabla U=0 \text { in } \Omega \backslash \bar{\Omega}_{\gamma}, \\
& U=r-\varepsilon \log \left(1+\left(u_{\varepsilon}-u_{\varepsilon}(0)\right) e^{r / \varepsilon}\right) \quad \text { on } \partial \Omega_{\gamma}, \\
& U=-\varepsilon \log \left(C_{\infty}+\varphi-u_{\varepsilon}(0)\right) \text { on } \partial \Omega .
\end{aligned}
$$

We want to estimate $\partial U / \partial n$ on $\partial \Omega$. Choose a function $\eta \in C^{2}(\Omega) \cap C^{1, \beta}(\bar{\Omega})$ satisfying

$$
-\Delta \eta=0 \quad \text { in } \Omega, \quad \eta=-\log \left(C_{\infty}+\varphi-u_{\varepsilon}(0)\right) \quad \text { on } \partial \Omega .
$$

Then $G=U-\varepsilon \eta$ satisfies

$$
\begin{aligned}
K_{\varepsilon} G & :=-\frac{\varepsilon}{2} \Delta G+|\nabla G|^{2} / 2-b \cdot \nabla G-\varepsilon \nabla G \cdot \nabla \eta / 2 \\
& =-\varepsilon^{2}|\nabla \eta|^{2} / 2+\varepsilon b \cdot \nabla \eta:=\varepsilon g_{\varepsilon} \quad \text { in } \Omega \backslash \bar{\Omega}_{\gamma}, \\
G= & 0 \quad \text { on } \partial \Omega, \\
G= & r+\varepsilon h_{\varepsilon} \quad \text { on } \partial \Omega_{\gamma},
\end{aligned}
$$


where $g_{\varepsilon}$ is bounded in $C(\bar{\Omega})$ and $h_{\varepsilon}$ is bounded in $C\left(\partial \Omega_{\gamma}\right)$. We are going to build a supersolution to (41). First of all let us define a function $c(y, s)$, $y \in \partial \Omega, s \geq 0$, as follows:

$$
\begin{aligned}
& c(y, 0)=-2 b \cdot n(y), \\
& c(y, s)=\operatorname{Min}_{z \in \partial \Omega}\left\{c(z, 0)+|z-y|^{2} /\left(2 s^{1 / 2}\right)\right\} \geq 0 .
\end{aligned}
$$

We give some properties of $c$ which are easy to check:

$$
\begin{gathered}
c(y, s) \leq \operatorname{Max}_{z \in \partial \Omega} c(z, 0), \quad \partial c(y, s) / \partial s \leq 0, \\
|\partial c(y, s) / \partial \chi| \leq C / s^{1 / 4} \quad \forall \chi,|\chi|=1, \chi \text { tangent to } \partial \Omega \text { at } y, \\
\partial^{2} c(y, s) / \partial \chi^{2} \leq 1 / s^{1 / 2} \quad \forall \chi,|\chi|=1, \chi \text { tangent to } \partial \Omega \text { at } y .
\end{gathered}
$$

For example, let us check (45): take $y^{\prime}, y^{\prime \prime}, y \in \partial \Omega, s \geq 0$ and denote $\underline{Y}$ a point of $\partial \Omega$ which realizes the minimum in (42). We have

$$
\begin{gathered}
c\left(y^{\prime}, s\right)+c\left(y^{\prime \prime}, s\right)-2 c(y, s) \leq\left(\left|\underline{Y}-y^{\prime}\right|^{2}+\left|\underline{Y}-y^{\prime \prime}\right|^{2}-2|\underline{Y}-y|^{2}\right) /\left(2 s^{1 / 2}\right) \\
\leq\left(-2\left(\underline{Y}-y, y^{\prime}+y^{\prime \prime}-2 y\right)+\left|y-y^{\prime}\right|^{2}+\left|y-y^{\prime \prime}\right|^{2}\right) /\left(2 s^{1 / 2}\right),
\end{gathered}
$$

choosing $y^{\prime}$ and $y^{\prime \prime}$ conveniently, i.e. $\left|y-y^{\prime}\right|=\left|y-y^{\prime \prime}\right|,\left|y^{\prime}+y^{\prime \prime}-2 y\right| \leq c\left|y-y^{\prime}\right|^{2}$, we obtain (45).

We denote now by $P x$ the projection of $x \in \bar{\Omega} \backslash \Omega_{\gamma}$ onto $\partial \Omega$, and we choose a modulus of continuity $\rho$ such that

$$
\rho(\gamma) \geq \gamma^{1 / 4}, \quad \rho(\gamma)+c(P x, d(x))+2 b \cdot n(P x) \geq \gamma^{1 / 4} \quad \text { on } \bar{\Omega} \backslash \Omega_{\gamma},
$$

this is possible since $c$ is continuous. We choose a supersolution to (41) as follows:

$$
\psi(x)=\int_{0}^{d(x)} c(P x, s) d s+\rho(\gamma) d(x) .
$$

Let us prove that $\psi$ is a supersolution. Thus, let $x_{0}$ be given and let us compute (41) in the local coordinates such that $e_{N}=-n\left(P x_{0}\right), e_{1}, \ldots, e_{N-1}$ are orthonormal to $e_{N}$. We have

$$
\frac{\partial \psi}{\partial i}=\int_{0}^{d(x)} \frac{\partial P}{\partial N} \nabla_{y} c(P x, s) d s+(\rho(\gamma)+c(P x, d(x))) \frac{\partial d(x)}{\partial i},
$$

and at $x_{0}$,

$$
\begin{aligned}
\frac{\partial^{2} \psi}{\partial i^{2}}= & \int_{0}^{d(x)}\left(\frac{\partial^{2} P}{\partial i^{2}} \nabla_{y} c(P x, s)+\Delta_{y} c(P x, d(x))\right) d s \\
& +(\rho(\gamma)+c(P x, d(x))) \frac{\partial^{2} d(x)}{\partial i^{2}} \\
& \frac{\partial^{2} \psi}{\partial N^{2}}=\frac{\partial c(P x, d(x))}{\partial s}+c(P x, d(x)) .
\end{aligned}
$$


Using (42)-(44), we obtain for some constant $C$ independent of $\varepsilon, \gamma$

$$
-\Delta \psi\left(x_{0}\right) \geq-C
$$

therefore

$$
\begin{aligned}
K_{\varepsilon} \psi\left(x_{0}\right) \geq & -C \varepsilon+\left(\rho(\gamma)+c\left(P x_{0}, d\left(x_{0}\right)\right)\right) \\
& \cdot\left(2 b \cdot n\left(P x_{0}\right)+\rho(\gamma)+c\left(P x_{0}, d\left(x_{0}\right)\right)\right) / 2-C d(x)^{3 / 4} .
\end{aligned}
$$

From the choice of $\rho(\gamma)$, this is larger than

$$
-C \varepsilon+\gamma^{1 / 2}-C \gamma^{3 / 4} \geq-C \varepsilon+\gamma^{1 / 2} / 2 \text { for } \gamma \text { small enough. }
$$

Therefore, choosing $r(\gamma) \leq \psi(\gamma), \psi$ is a supersolution of (41), hence $\psi \geq G$ and

$$
\partial G(x) / \partial n \geq-c(x, 0)-\rho(\gamma)=2 b \cdot n(x)-\rho(\gamma) \text { on } \partial \Omega,
$$

i.e.

$$
\partial U(x) / \partial n \geq 2 b \cdot n(x)-\rho(\gamma)-C \varepsilon \text { on } \partial \Omega,
$$

i.e.

$$
\varepsilon \partial u_{+}(x) / \partial n \leq-2 b \cdot n(x)\left(\varphi-u_{\varepsilon}(0)+C_{\infty}\right)+C \varepsilon+C \rho(\gamma) \text { on } \partial \Omega .
$$

To prove the other inequality we are going to build a subsolution of (41), this is more difficult because the limit equation of (41) does not admit any strict subsolution if $b=0$ at some point of $\partial \Omega$. That is the reason why we need to assume that

$$
\exists \nu, \quad-b \cdot n \geq \nu>0 \text { on } \partial \Omega .
$$

As before let us define a function $a(y, s), y \in \partial \Omega, s \geq 0$, as

$$
\begin{aligned}
& a(y, 0)=-2 b \cdot n(y), \\
& a(y, s)=\underset{z \in \partial \Omega}{\operatorname{Max}} a(z, 0)-\varepsilon^{2}|z-y|^{2} /\left(2 s^{1 / 2}\right) \geq 0 .
\end{aligned}
$$

It satisfies

$$
\begin{aligned}
& a(y, s) \geq \underset{z \in \partial \Omega}{\operatorname{Min}} a(z, 0), \quad \partial a(y, s) / \partial s \geq 0, \\
& |\partial a(y, s) / \partial \chi| \leq C \varepsilon / s^{1 / 4} \quad \forall \chi,|\chi|=1, \chi \text { tangent to } \partial \Omega \text { at } y, \\
& \partial^{2} a(y, s) / \partial \chi^{2} \geq \varepsilon^{2} / s^{1 / 2} \quad \forall \chi,|\chi|=1, \chi \text { tangent to } \partial \Omega \text { at } y .
\end{aligned}
$$

As before we choose $\rho(\gamma)$ so that

$$
\begin{aligned}
-\rho(\gamma)+b(x) \cdot n(P x)+a(P x, d(x)) / 2 \leq-2 \gamma, \quad a(P x, d(x))-\rho(\gamma) & \geq \nu, \\
\rho(\gamma) & \rightarrow 0 \text { as } \gamma \rightarrow 0 .
\end{aligned}
$$

Now, we choose a function $\zeta \in C^{2}\left(\bar{\Omega}_{\gamma}\right)$, with $\zeta=1, \nabla \zeta=0$ on $\partial \Omega$, and $\zeta=0$ for $d(x) \geq r(\gamma) /\left(6\|b\|_{\infty}\right) \quad(r(\gamma)$ has been fixed before to build the supersolution), we also assume that $\|\zeta\|_{C} 1 \leq C / r$ and $\|\zeta\|_{C}{ }^{2} \leq C / r$. We define

$$
H=U-\varepsilon \eta \zeta \text {, }
$$


and

$$
\begin{aligned}
\underline{K}_{\varepsilon} H & :=\frac{\varepsilon}{2} \Delta H+|\nabla H|^{2} / 2-b \cdot \nabla H-\varepsilon \nabla H \cdot \nabla(\zeta \eta) \\
& \geq-C \varepsilon / r(\gamma) 1_{\left\{x ; d(x) \leq r(\gamma) /\left(3\|b\|_{\infty}\right)\right\} \quad(\varepsilon \ll r) \text { on } \Omega \backslash \bar{\Omega}_{\gamma},} \\
H= & 0 \text { on } \partial \Omega, \\
H= & +\varepsilon h_{\varepsilon} \text { on } \partial \Omega_{\gamma} .
\end{aligned}
$$

We define now

and

$$
\psi(x)=\int_{0}^{d(x)} a(P x, s) d s-\rho(\gamma) d(x)
$$

$$
\Psi(x)=-\varepsilon \log \left(e^{-\psi / \varepsilon}+e^{-r /(2 \varepsilon)}\right) .
$$

A simple computation shows that

$$
\underline{K}_{\varepsilon} \Psi:=e^{-\psi / \varepsilon} /\left(e^{-\psi / \varepsilon)}+e^{-r /(2 \varepsilon)}\right)\left\{-\frac{\varepsilon}{2} \Delta \psi+|\nabla \psi|^{2} / 2-(b+\varepsilon \nabla(\zeta \eta)) \cdot \nabla \psi\right\} .
$$

In order to estimate this, we follow the previous computation of $\nabla \psi, D^{2} \psi$. We have at $x_{0}$

$$
\begin{gathered}
\partial \psi / \partial i=\int_{0}^{d(x)} \partial P / \partial i \nabla_{y} a(P x, s) d s, \quad i<N, \\
\partial \psi / \partial N=a(P x, d(x))-\rho(\gamma), \\
\partial^{2} \psi / \partial i^{2}=\int_{0}^{d(x)}\left(\partial^{2} P / \partial i^{2} \nabla_{y} a(P x, s)+\Delta_{y} a(P x, d(x))\right) d s \\
+\rho(\gamma) \partial^{2} d / \partial i^{2}, \quad i<N, \\
\partial^{2} \psi / \partial N^{2}=\partial a(P x, d(x)) / \partial s .
\end{gathered}
$$

Therefore,

$$
\begin{aligned}
-\frac{\varepsilon}{2} \Delta \psi & +|\nabla \psi|^{2} / 2-(b+\varepsilon \nabla(\zeta \eta)) \cdot \nabla \psi \\
\leq & C \varepsilon / r+(a(P x, d(x))-\rho(\gamma)) \\
& \cdot[(b \cdot n+\varepsilon \nabla(\zeta \eta)) \cdot n(P x)+a(P x, d(x)) / 2-\rho(\gamma) / 2] \\
\leq & C \varepsilon / r-\nu \cdot(2 \gamma-C \varepsilon) .
\end{aligned}
$$

For $d(x) \leq r(\gamma) /\left(6\|b\|_{\infty}\right)$, we have

$$
\psi(x) \leq 3\|b\|_{\infty} d(x)<r(\gamma) / 2,
$$

i.e.

$$
\underline{K}_{\varepsilon} \Psi \leq-\gamma \nu / 2 \text { (for } \varepsilon, \gamma \text { small enough). }
$$

On $\Omega \backslash \bar{\Omega}_{\gamma}$, we have

$$
\underline{K}_{\varepsilon} \Psi \leq 0
$$

Since $\Psi \leq H$ on $\partial \Omega_{\gamma}, \Psi$ is less than $H$ everywhere and we conclude as before that

$$
\varepsilon \partial u_{+}(x) / \partial n \geq-2 b \cdot n(x)\left(\varphi-u_{\varepsilon}(0)+C_{\infty}\right)+C \varepsilon+C \rho(\gamma) \quad \text { on } \partial \Omega .
$$


Performing the same analysis on $u_{-}$, this shows formula (7) and concludes the proof of Theorem 3 because the method of Matkowsky and Schuss presented in the introduction allows us to compute the relation between $u_{\varepsilon}(0)$ and $\mu_{\varepsilon}$.

\section{APPENDIX 1. ThE NOTION OF VISCOSITY SOLUTIONS TO HAMILTON-JACOBI EQUATIONS}

The notion of viscosity solution was introduced by Crandall-Lions to give a sense and a uniqueness criteria to H.J. equations

$$
H(x, u, \nabla u)=0 \text { in } \Omega .
$$

Then, to solve state constraints problems, Soner extended this notion to give a sense to the boundary condition

$$
H(x, u, \nabla u) \geq 0 \text { on } \partial \Omega .
$$

These definitions of viscosity solutions can be given for discontinuous solutions of (54), (55) although general uniqueness results only hold for continuous solutions (and with good properties of $H$ ). However, it is possible to use discontinuous viscosity solutions as we did in Theorem 2.

First, let us recall the definition of viscosity solutions.

Definition 1. An upper semicontinuous (on $\bar{\Omega}$ ) function $\bar{u}$ is called a viscosity subsolution of (54) if, for any $\phi \in C^{2}(\Omega)$ and $x_{0} \in \Omega$ such that

$$
\operatorname{Max}_{\bar{\Omega}}(\bar{u}-\phi)=(\bar{u}-\phi)\left(x_{0}\right) \text {, }
$$

then

$$
H\left(x_{0}, \bar{u}\left(x_{0}\right), \nabla \phi\left(x_{0}\right)\right) \leq 0 .
$$

We say that $\partial u / \partial n \leq \gamma$ on $\partial \Omega$, if, when the above maximum is attained for some $x_{0} \in \partial \Omega$, then

$$
\operatorname{Min}\left(H\left(x_{0}, \bar{u}\left(x_{0}\right), \nabla \phi\left(x_{0}\right)\right), \partial \phi\left(x_{0}\right) / \partial n-\gamma\left(x_{0}\right)\right) \leq 0 .
$$

Let us point out that this convention for the Neumann case differs from the one in [22, 24], our definition is fitting with the Dirichlet case $[17,2,3]$.

Definition 2. A lower semicontinuous (on $\bar{\Omega}$ ) function $\underline{u}$ is called a viscosity supersolution of (54)-(55) if, for any $\phi \in C^{2}(\bar{\Omega})$ and $x_{0} \in \bar{\Omega}$ such that

$$
\operatorname{Min}(\underline{u}-\phi)=(\underline{u}-\phi)\left(x_{0}\right),
$$

then

$$
H\left(x_{0}, \underline{u}\left(x_{0}\right), \nabla \phi\left(x_{0}\right)\right) \geq 0 .
$$

Of course to consider supersolutions of (54), we have to replace $x_{0} \in \bar{\Omega}$ by $x_{0} \in \Omega$ above. The case of Neumann conditions is given in $\S I I$ and is an obvious extension of these definitions. We refer to [7] for the motivations, various extensions of these definitions, and for the relations with optimal control problems. 
It is easy to see on this definition that 0 is not a supersolution on the boundary of $(5)-\left(5^{\prime}\right)$. Indeed, $0+\lambda d(x)$ attains a minimum point at any point of $x \in \partial \Omega$, for any $\lambda>0$, but for $\lambda$ small enough, we do not have

$$
H(x,-\lambda \nabla d(x))=\lambda^{2}+\lambda b(x) \cdot n(x) \geq 0,
$$

at points such that $b(x) \cdot n(x)<0$.

$$
\text { Appendix 2. Proof of }(\mathrm{H} 3)+(\mathrm{H} 1) \Rightarrow(\mathrm{H} 2)
$$

Let us consider the function defined for $x \in \bar{\Omega} \backslash B_{\delta}$ by

$$
\begin{aligned}
\phi_{\alpha}(x)=\inf \left\{\int_{0}^{\tau}|\dot{y}+b(y)|^{2} / 2 d s-\alpha \tau ; y(0)=x, y \in H^{1}(0, \tau),\right. \\
\left.y(s) \in \bar{\Omega} \backslash B_{\delta} \forall s \leq \tau \leq+\infty, y(\tau) \in \partial B_{\delta} \text { if } \tau<+\infty\right\} .
\end{aligned}
$$

Let us show that $\phi_{\alpha}$ remains bounded for $\alpha$ small enough.

Assume, by contradiction, that for some $\alpha_{n}>0, \alpha_{n} \rightarrow 0$, there exists $x_{\alpha_{n}}, \tau_{\alpha_{n}}$ with (dropping the index $n$ )

$$
\left\{\int_{0}^{\tau_{\alpha}}\left|\dot{y}_{x_{\alpha}}+b\right|^{2} / 2 d s-\alpha \tau_{\alpha} \leq-1, y_{x}(0)=x_{\alpha}, \forall t \leq \tau_{\alpha} y_{x}(t) \in \bar{\Omega} \backslash B_{\delta}\right\} .
$$

Then, there exists $s_{\alpha}$ such that $s_{\alpha}+T \leq \tau_{\alpha}$ and

$$
\int_{s_{\alpha}}^{s_{\alpha}+T}\left|\dot{y}_{x_{\alpha}}+b\right|^{2} / 2 d s-\alpha T \leq 0 \quad \forall \alpha<1 / T,
$$

where $T$ denotes the time for which any solution of

$$
\dot{y}=b(y), \quad y(0) \in x,
$$

has left $\bar{\Omega} \backslash B_{\delta}$ as is assumed in (H3).

Let us prove (57). If we had

$$
\int_{n T}^{(n+1) T}\left|\dot{y}_{x_{\alpha}}+b\right|^{2} / 2 d s-\alpha T>0 \text { for any } n \geq 0 \text { with }(n+1) T \leq \tau_{\alpha},
$$
then,

$$
\int_{0}^{\tau_{\alpha}}\left|\dot{y}_{x_{\alpha}}+b\right|^{2} / 2 d s-\alpha \tau_{\alpha} \geq \int_{T E\left(\tau_{\alpha} / T\right)}^{\tau_{\alpha}}\left(\left|\dot{y}_{x_{\alpha}}+b\right|^{2} / 2-\alpha\right) d s \geq-\alpha T,
$$

where $E(r)$ denotes the entire part of $r$. This is a contradiction for $\alpha<1 / T$. Thus (57) holds.

Now, setting $z_{\alpha}=y_{x_{\alpha}}\left(s_{\alpha}\right)$, we may extract a subsequence such that $z_{\alpha} \rightarrow z_{0}$ and (57) shows that

$$
\int_{0}^{T}\left|\dot{y}_{\alpha}+b\left(y_{\alpha}\right)\right|^{2} / 2 d s \leq T \alpha,
$$

where $y_{\alpha}(t)=y_{x_{\alpha}}\left(s_{\alpha}+t\right)$. Therefore $\dot{y}_{\alpha}$ is bounded in $L^{2}[0, T]$ and thus, extracting again a subsequence,

$$
y_{\alpha}(t) \rightarrow y_{0}(t) \text { uniformly on }[0, T] .
$$


$y_{0}(t)$ satisfies

$$
\int_{0}^{T}\left|\dot{y}_{0}+b\left(y_{0}\right)\right|^{2} / 2 d s=0
$$

i.e.

$$
\dot{y}_{0}=-b\left(y_{0}\right), \quad y_{0}(0)=z_{0}, \quad y(s) \in \bar{\Omega}, \quad 0 \leq s \leq T .
$$

This contradicts the definition of $T$ and shows that

$$
\phi_{\alpha}(x) \geq-1 \text { for } \alpha \text { small enough. }
$$

Remark. This proof is close to the one in $[9,26]$.

It is easy to check that $\phi_{\alpha}$ is Lipschitz continuous and by classical arguments, it is a viscosity solution of

$$
\begin{array}{ll}
H\left(x, \nabla \phi_{\alpha}\right)=-\alpha & \text { in } \Omega \backslash \bar{B}_{\delta}, \\
\phi_{\alpha}=0 & \text { on } \partial B_{\delta} .
\end{array}
$$

$\phi_{\alpha}$ also satisfies the state constraints boundary condition on $\partial \Omega$, but we have to see that it implies the Neumann condition for subsolutions, i.e.

$$
\partial \phi_{\alpha} / \partial n \leq-2 b \cdot n \text { on } \partial \Omega \text {. }
$$

To do so, let us rewrite (56) as a control problem,

$$
\begin{gathered}
\left\{\begin{array}{l}
\dot{y}(t)=v(t), \quad v(t) \in L^{\infty}\left(\mathbf{R}^{+} ; \mathbf{R}^{N}\right), \\
y(0)=x,
\end{array}\right. \\
\phi_{\alpha}(x)=\inf _{A}\left\{\int_{0}^{\tau}|v(s)+b(y(s))|^{2} / 2 d s-\alpha \tau\right\},
\end{gathered}
$$

where

$$
A=\left\{v(\cdot) \in L^{\infty}\left(\mathbf{R}^{+} ; \mathbf{R}^{N}\right), y(s) \in \bar{\Omega} \backslash B_{\delta}, \forall s \leq \tau \leq+\infty, y(\tau) \in \partial B_{\delta} \text { if } \tau<+\infty\right\} .
$$

Notice that the constraints $y \in H^{1}$ or $\dot{y}$ bounded are equivalent because the Hamiltonian $H$ may be written

$$
H(x, p)=\sup _{v \in \mathbf{R}^{N}}\left\{-v \cdot p-|v+p|^{2} / 2\right\}=\sup _{|v| \leq|b|+2|p|}\left\{-v \cdot p-|v+p|^{2} / 2\right\},
$$

and $\nabla \phi_{\alpha}$ (or $p$ in the above formula) is a priori bounded. (59)-(60) are equivalent to

$$
\begin{gathered}
\left\{\begin{array}{l}
d y(t)=v(t) d t+d A(t), \quad v(t) \in L^{\infty}\left(\mathbf{R}^{+} ; \mathbf{R}^{N}\right), \\
y(0)=x, \quad A(t)=-\int_{0}^{T} 1_{y(s) \in \partial \Omega}[v(s) \cdot n(y(s))]^{+} n(y(s)) d s,
\end{array}\right. \\
\phi_{\alpha}(x)=\inf _{B}\left\{\int_{0}^{T}\left|v+b(y)-(v \cdot n)^{+} 1_{y \in \partial \Omega} n\right|^{2} / 2 d s-\alpha \tau\right\}, \\
B=\left\{v(\cdot) \in L^{\infty}\left(\mathbf{R}^{+} ; \mathbf{R}^{N}\right), y(\tau) \in \partial B_{\delta} \text { if } \tau<+\infty\right\} .
\end{gathered}
$$

And the quantity under the integral in (62) is also

$$
|v+b(y)|^{2} / 2 d s+d|A|(s)\left[-b \cdot n+(v \cdot n)^{+} / 2-v \cdot n\right]
$$


$(d|A|$ denotes the total variation of the measure with bounded variations $d A)$. (61)-(62) represent an optimal control problem for a reflected process, the cost of the reflection is

$$
\gamma(y, v)=-b \cdot n+(v \cdot n)^{+} / 2-v \cdot n,
$$

and when $d|A|(s) \neq 0, v \cdot n$ is positive, therefore

$$
\gamma(y, v) \leq b \cdot n \leq-2 b \cdot n \quad(b \cdot n \leq 0 \text { by }(\mathrm{H} 3)),
$$

and thus $\phi_{\alpha}$ satisfies

$$
\partial \phi_{\alpha} / \partial n \leq-2 b \cdot n \text { on } \partial \Omega
$$

and $(\mathrm{H} 2)$ is proved.

\section{REFERENCES}

1. M. Bardi, An asymptotic formula for the Green's function of an elliptic operator, Ann. Scuola Norm. Sup. Pisa Cl. Sci. (4) 14 (1987), 569-612.

2. G. Barles and B. Perthame, Discontinuous solutions of deterministic optimal stopping time problems, Math. Methods and Numer. Anal. 21 (1987), 557-579.

3. __ Exit time problems in optimal control and vanishing viscosity method, SIAM J. Control Optim. 26 (1988).

4. A. Bensoussan, Méthodes de perturbations singulières, Masson, Paris, 1989.

5. A. Bensoussan and J. L. Lions, On the asymptotic behavior of the solution of variational inequalities, Theory of Nonlinear Operators, Akademie-Verlag, Berlin, 1978.

6. I. Capuzzo-Dolcetta and P. L. Lions, State constraints problem for Hamilton-Jacobi equations, Trans. Amer. Math. Soc. (to appear).

7. M. G. Crandall and P. L. Lions, Viscosity solutions of Hamilton-Jacobi equations, Trans. Amer. Math. Soc. 177 (1983), 1-42.

8. M. Day, Recent progress on the small parameter exit problem, preprint.

9. L. C. Evans and H. Ishii, A pde approach to some asymptotic problems concerning random differential equations with small noise intensities, Ann. Inst. H. Poincaré, Anal. Non Linéaire 2 (1985), 1-20.

10. L. C. Evans and P. E. Souganidis, A pde approach to geometric optics for certain semilinear parabolic equations, Indiana Univ. Math. J. 38 (1989), 141-172.

11. _ A pde approach to certain large deviation problems for systems of parabolic equations, preprint.

12. W. H. Fleming, Exit probabilities and optimal stochastic control, Appl. Math. Optim. 4 (1978), 329-346.

13. W. H. Fleming and P. E. Souganidis, Pde-viscosity solution approach to some problems of large deviations, Ann. Scuola Norm. Sup Pisa Cl. Sci. (4) 13 (1986), 71-108.

14. M. I. Freidlin and A. D. Wentzell, Random perturbations of dynamical systems, Springer, New York, 1984.

15. D. Gilbarg and N. S. Trudinger, Elliptic partial differential equations of second order, 2nd ed., Springer-Verlag, 1983.

16. H. Ishii, A simple direct proof of uniqueness for solutions of the Hamilton-Jacobi equations of Eikonal type, Proc. Amer. Math. Soc. 100 (1987), 247-251.

17. _ A boundary value problem of the Dirichlet type for Hamilton-Jacobi equations, preprint.

18. H. Ishii and P. L. Lions, Viscosity solutions of fully nonlinear second order elliptic PDEs, preprint. 
19. S. Kamin, Elliptic perturbation of a first order operator with a singular point of attracting type, Indiana Univ. Math. J. 27 (1978), 935-952.

20. __ Elliptic perturbation for linear and nonlinear equations with a singular point, preprint.

21. P. L. Lions, Optimal control of diffusion processes and Hamilton-Jacobi equations. Part II: Viscosity solutions and uniqueness, Comm. Partial Differential Equations 8 (1983), 12291276.

22. __ Neumann type boundary conditions for Hamilton-Jacobi equations, Duke Math. J. 52 (1985) 793-820.

23. B. J. Matkowsky and Z. Schuss, The exit problem for randomly perturbed dynamical systems, SIAM J. Appl. Math. 36 (1979), 365-382.

24. B. Perthame and R. Sanders, The Neumann problem for nonlinear second order singular perturbation problems, SIAM J. Math. Anal. 19 (1988), 295, 311.

25. M. H. Soner, Optimal control problems with state space constraints, Parts 1 and 2, SIAM J. Control Optim. 24(1) (1986), 1110-1122 and 24(2) (1986), 552-561.

26. A. D. Wentzell and M. I. Freidlin, On small perturbations of dynamical systems, Uspekhi Mat. Nauk. 25 (1970), 3-55; English transl., Russian Math. Surveys 25 (1970), 1-56.

Centre de Mathematiques Appliquées, Ecole Normale Supérieure, 75230 Paris Cedex 05, FRANCE

Current address: Département de Mathématiques, Université d'Orléans, BP 6749, 45067 Orléans Cx 2, France 